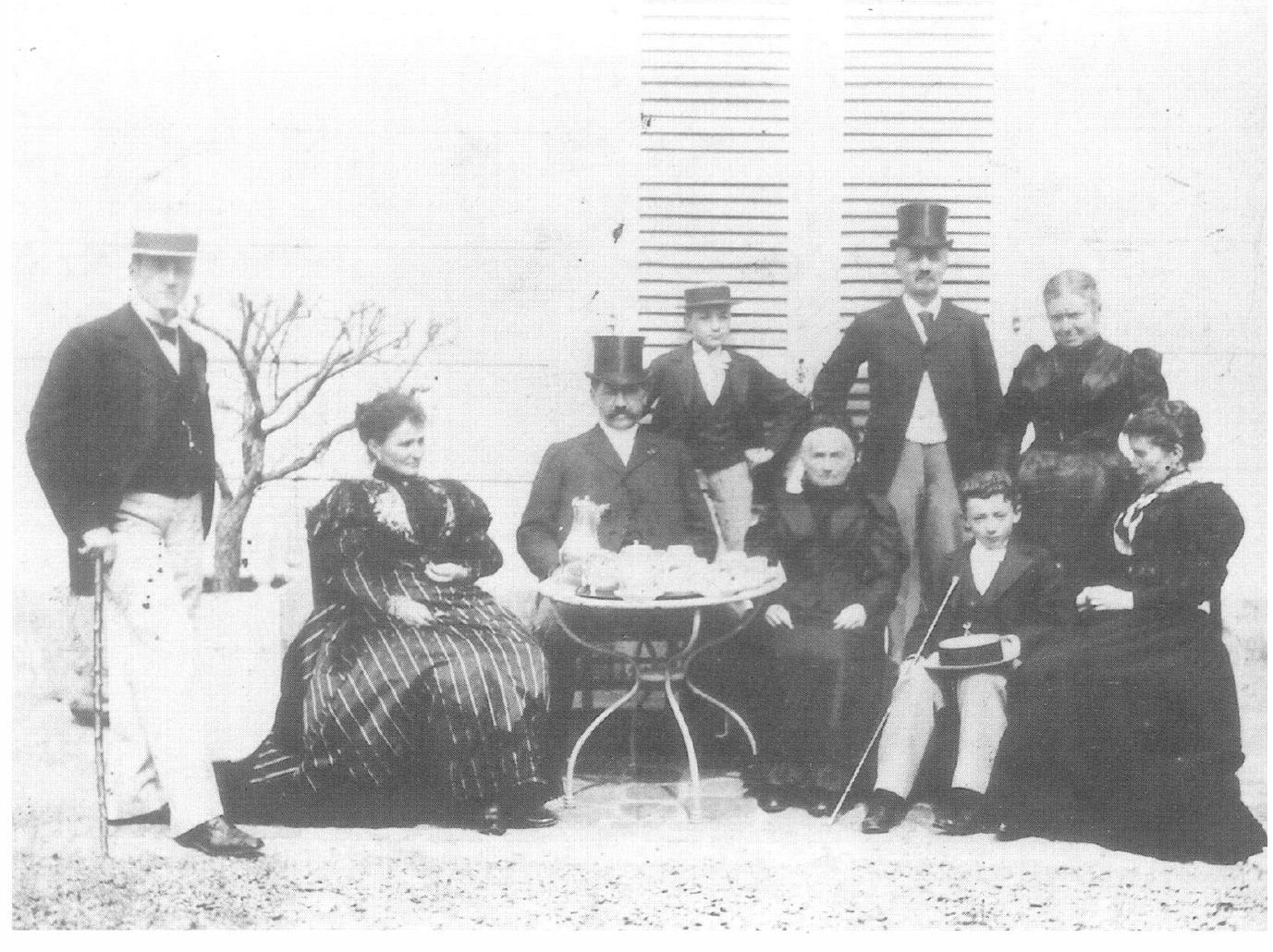

Ein Familienbild. Stehend vor der Fassade: Vater Alexandre und Bruder Albert, sitzend rechts die Mutter Amélie, neben ihr Paul Reynaud. Um 1890.

Privatbesitz Madame Evelyne Demey 


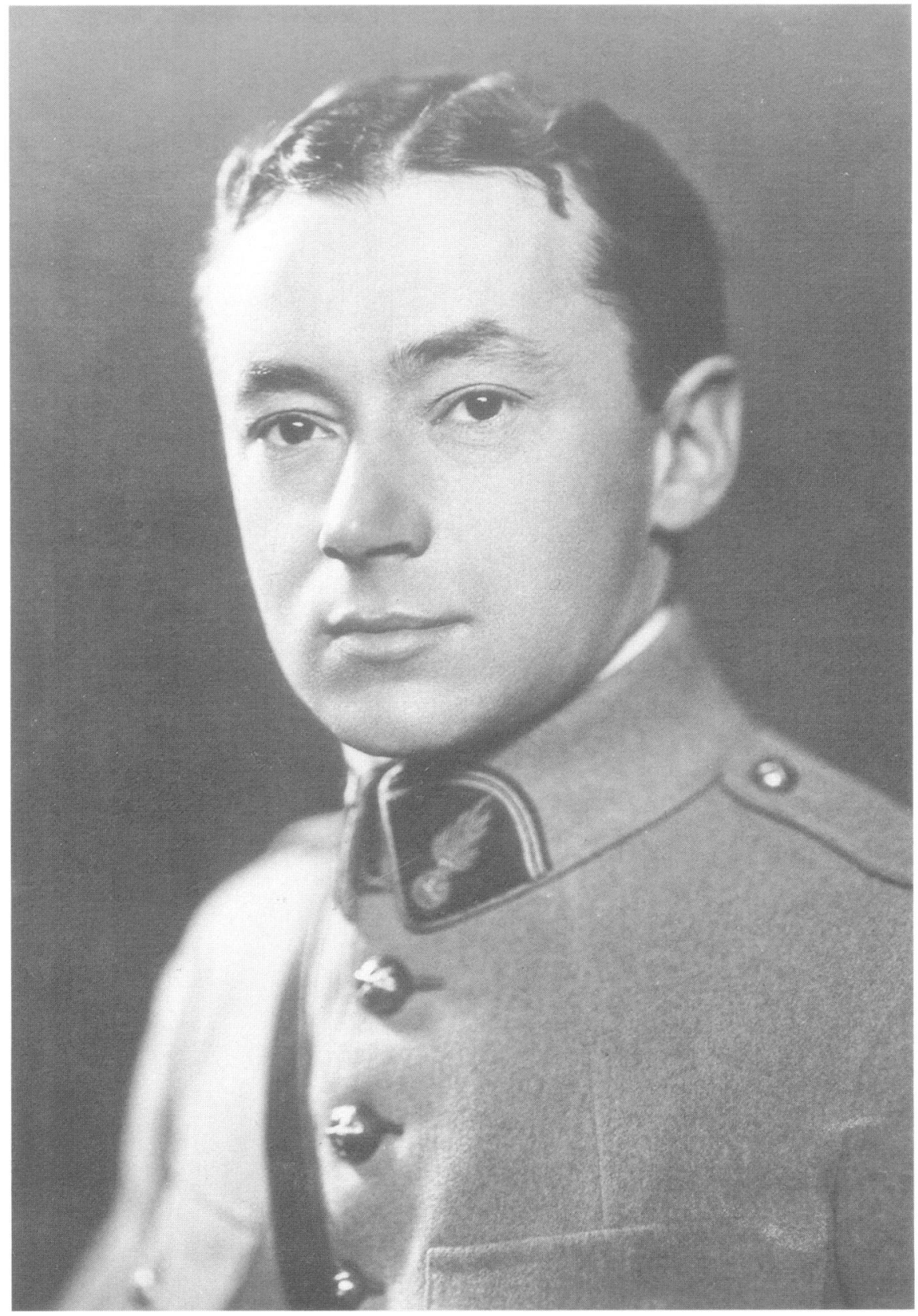

Als Soldat im Ersten Weltkries.

service listorigue de l'Armede derre, Vincennes 


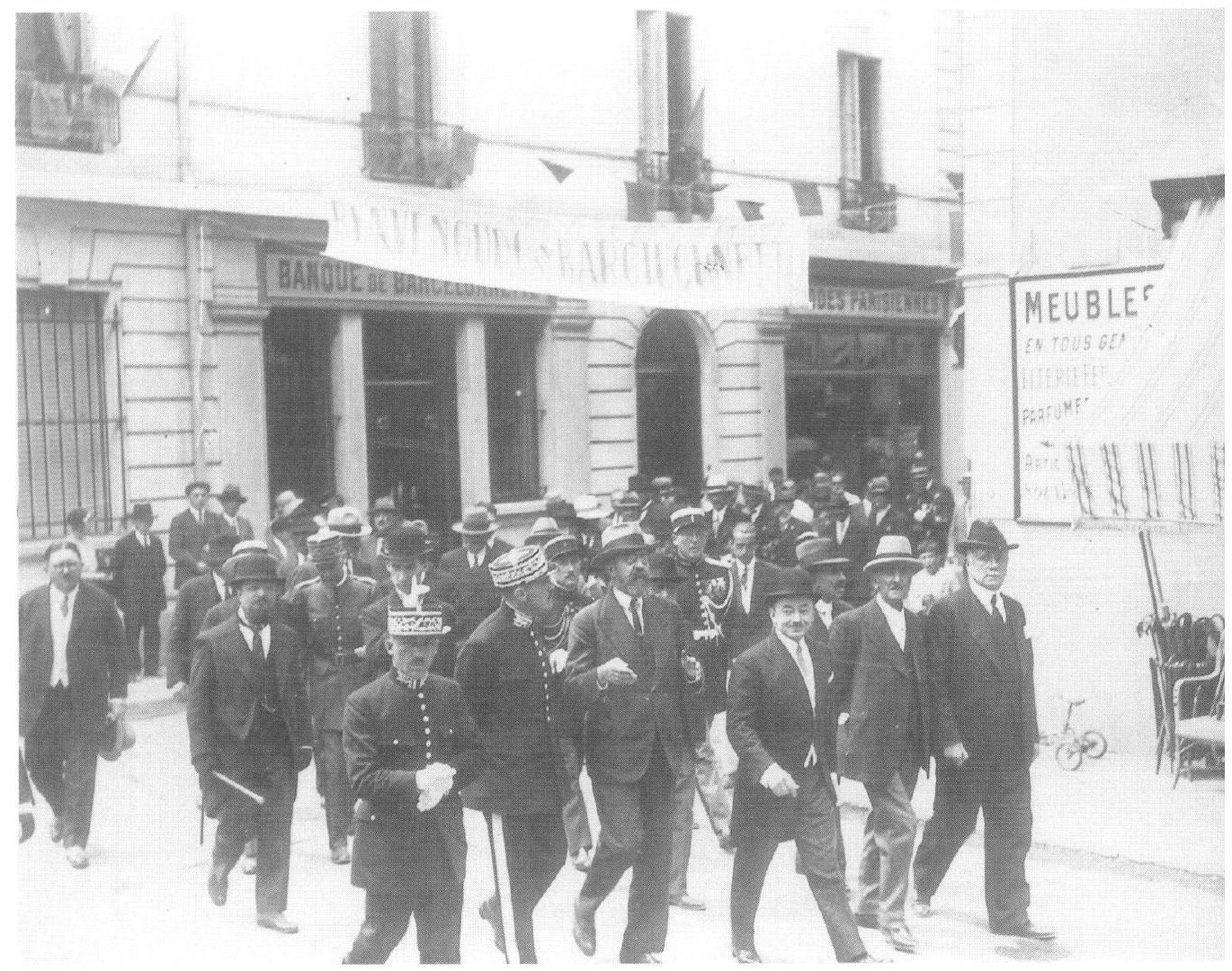

Beim Festzug aus Anlaß des 700. Jahrestags der Gründung von Barcelonnette. Reynaud wird flankiert von Senator André Honnorat (links), Bürgermeister Rémy Reynaud und Conseiller Général Paul Gassier (rechts), August 1931. Musée de la Vallée, Barcelonnette 


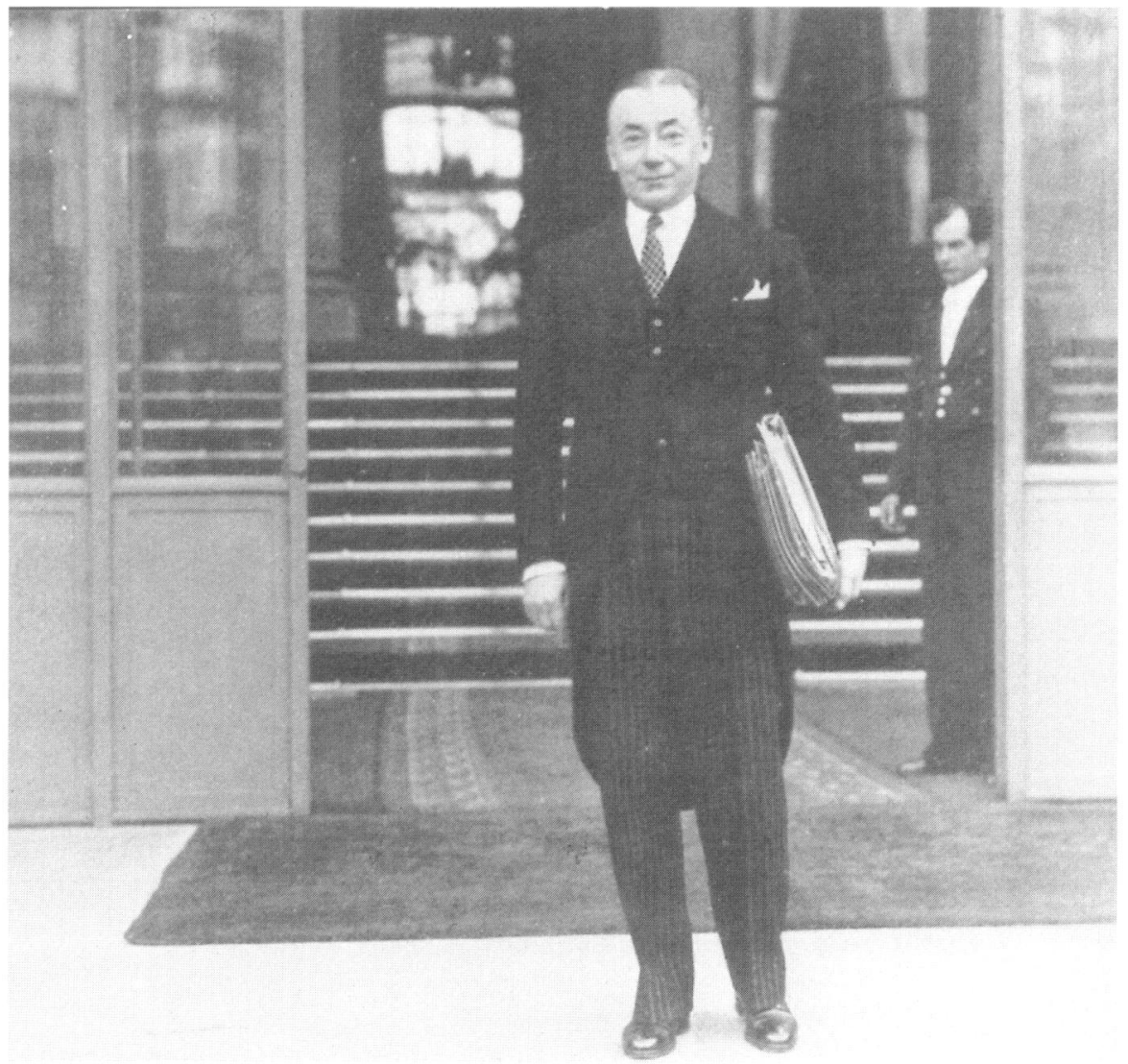

Revnaud verläßt den Élysćc-Palast, nachdem Staatspräsident Lebrun seine „Décrets-lois" zur Neuorientierung der Wirtschafts- und Finanzpolitik unterzeichnet hat, 12. November 1938.

Flammarion, Paris 


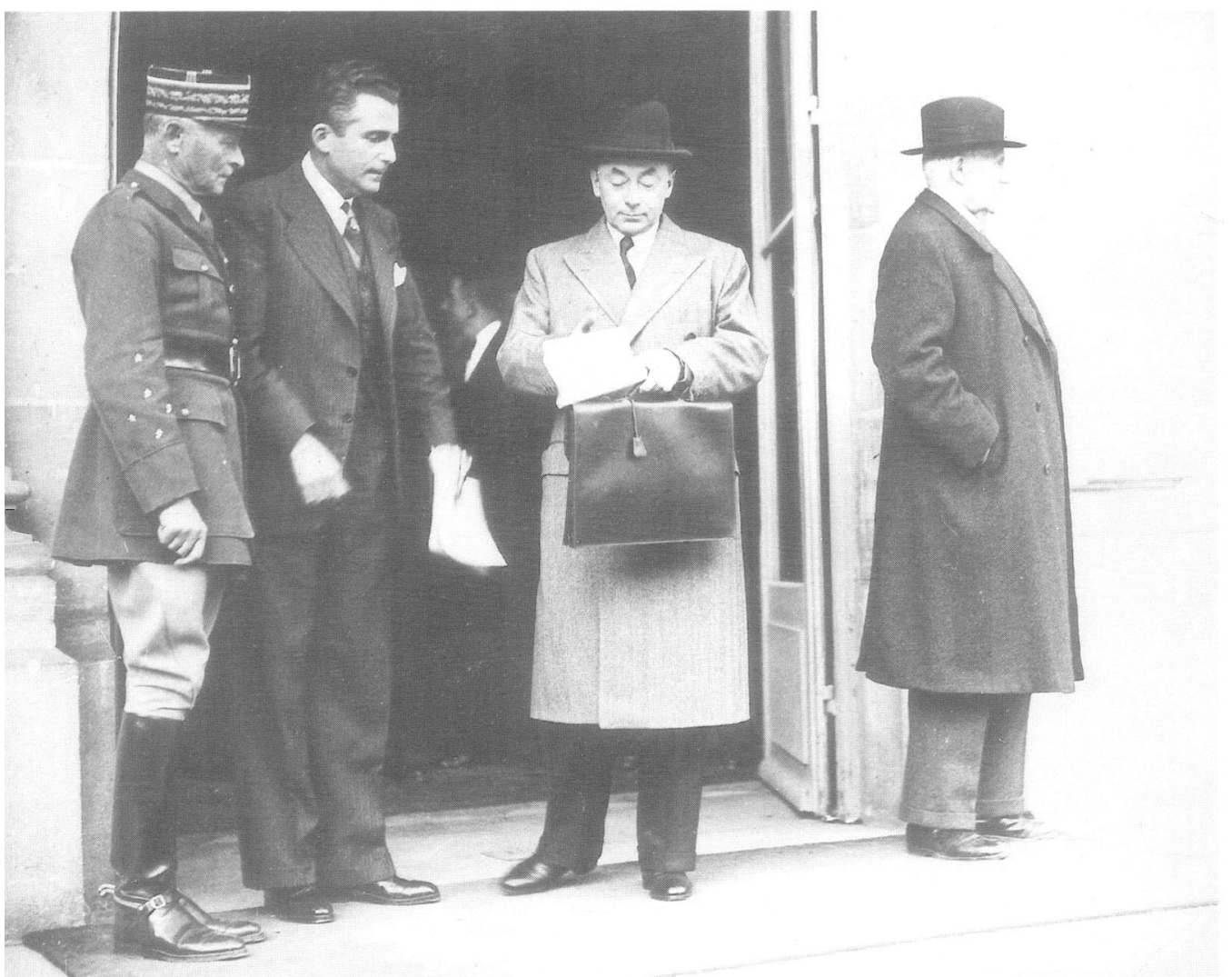

Nach Ende ciner Besprechung am 21. Mai 1940. Von links nach rechts: General Weygand, Paul Baudouin, Paul Reynaud, Marschall Pétain.

Agence Keystone, Paris 


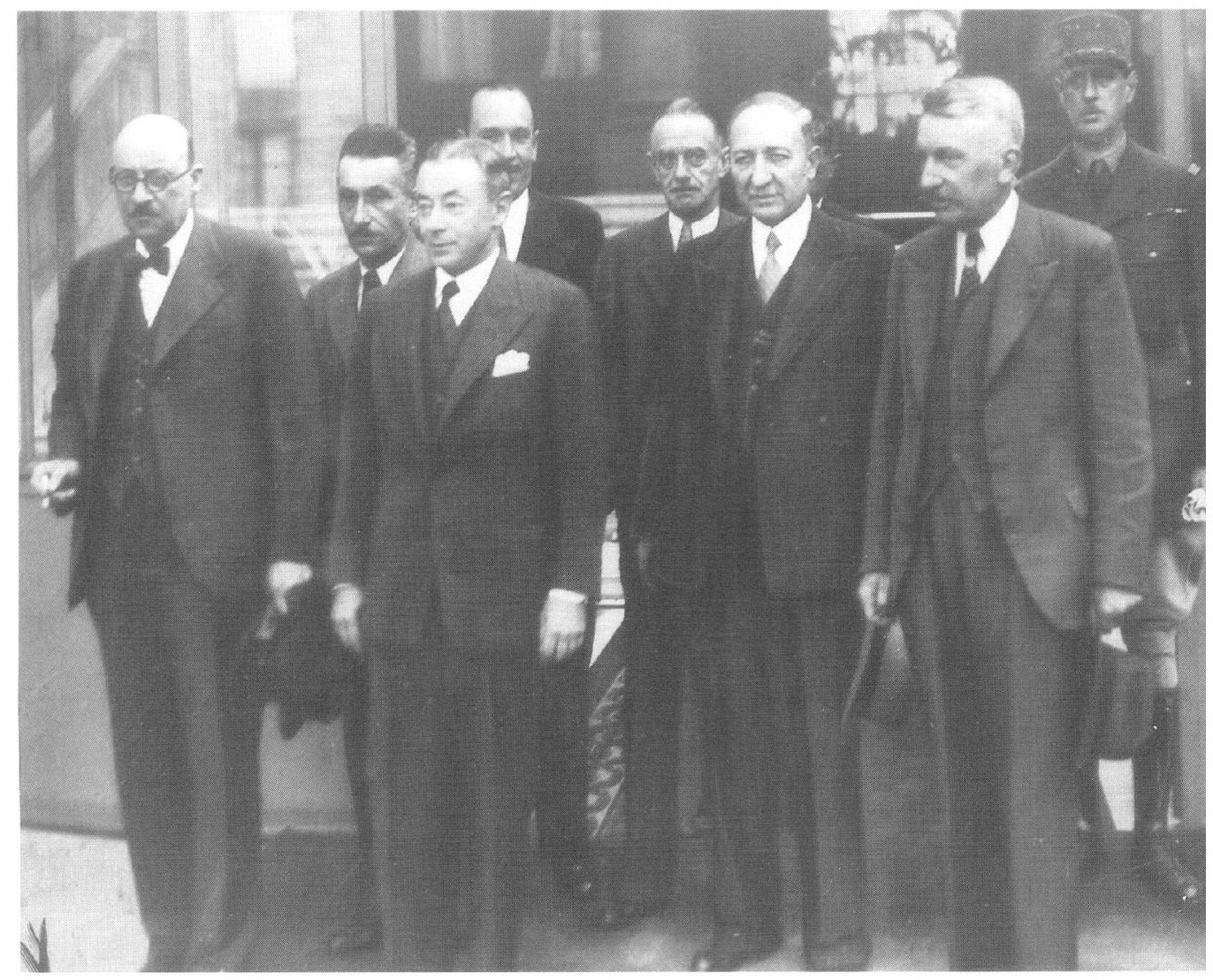

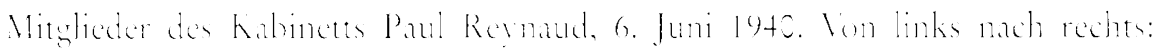

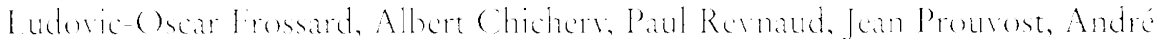

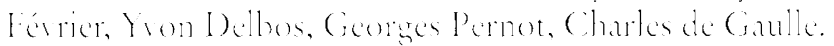

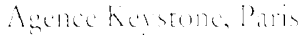




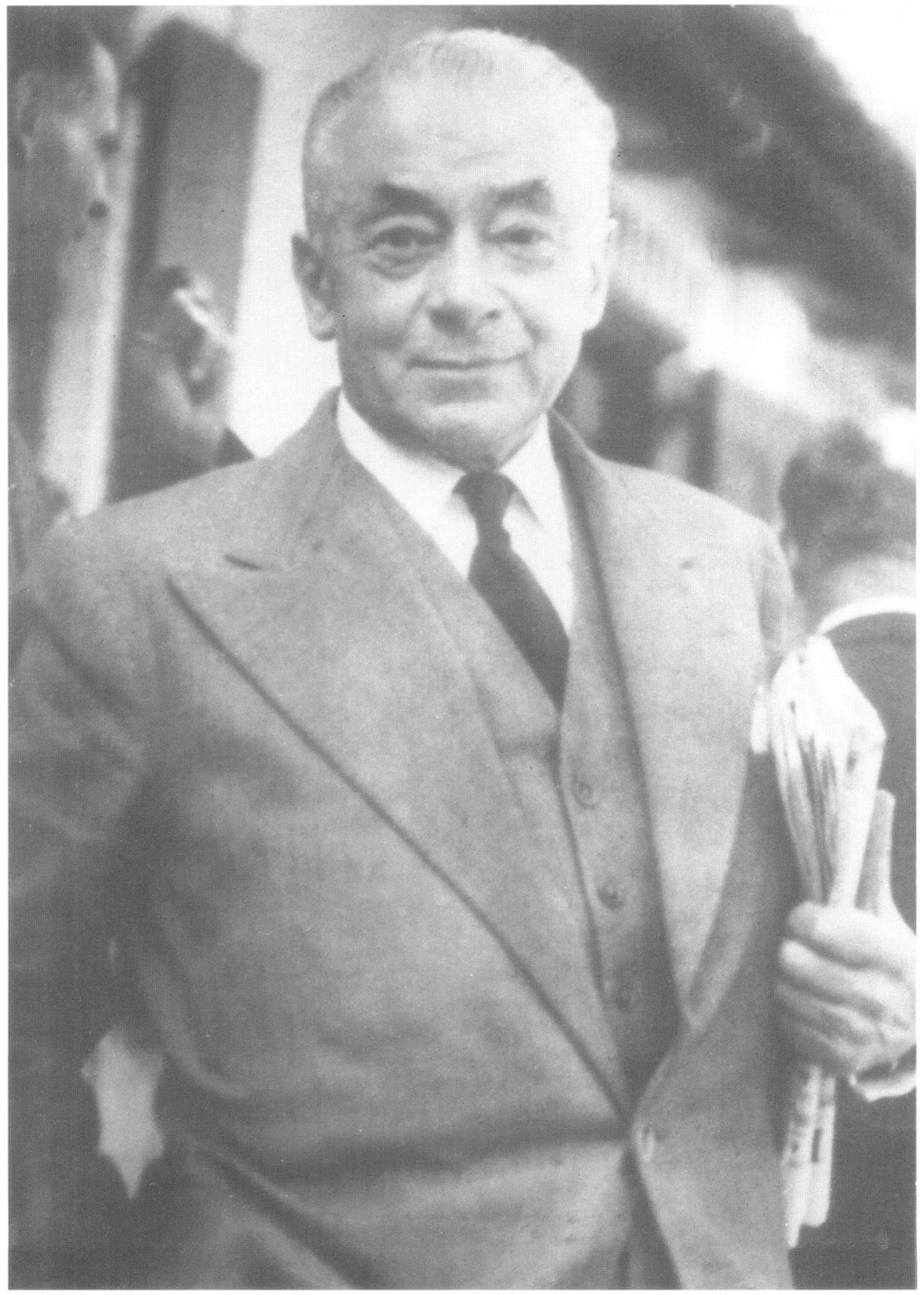

Paul Revnaud in seinen letyen Lebensiahren.

Privatbesity Madame Frelone I bemes 


\section{Abkürzungsverzeichnis}

AD Haute-Provence Archives Départementales des Alpes-de-Haute-Provence, Digne

AD Hautes-Alpes Archives Départementales des Hautes-Alpes, Gap

ADAP

AEF

AN

BAK

BDIC

$\mathrm{BN}$

CAD/Nantes

CAOM

CGT

DBFP

DDF

Euratom

EVG

EWG

FRUS

GWU

HEC

$\mathrm{HZ}$

JO

MAE

MRP

PA-AA

PCF

SFIO

SHAT

URD

$\mathrm{VfZ}$
Akten zur Deutschen Auswärtigen Politik

Archives du ministère des Finances, Paris

Archives Nationales, Paris

Bundesarchiv Koblenz

Bibliothèque de Documentation Internationale

Contemporaine, Nanterre

Bibliothèque Nationale, Paris

Centre des Archives Diplomatiques de Nantes

Centre des Archives d'Outre-Mer, Aix-en-Provence

Confédération Générale du Travail

Documents on British Foreign Policy

Documents Diplomatiques Français

Europäische Atomgemeinschaft

Europäische Verteidigungsgemeinschaft

Europäische Wirtschaftsgemeinschaft

Foreign Relations of the United States

Geschichte in Wissenschaft und Unterricht

Ecole des Hautes Etudes Commerciales, Jouy-en-Josas

Historische Zeitschrift

Journal Officiel de la République française

Ministère des Affaires Etrangères, Paris

Mouvement Républicain Populaire

Politisches Archiv des Auswärtigen Amts, Bonn

Parti Communiste Français

Section Française de l'Internationale Ouvrière

Service Historique de l'Armée de Terre, Vincennes

Union Républicaine Démocratique

Vierteljahrshefte für Zeitgeschichte 


\title{
Quellen- und Literaturverzeichnis
}

\author{
I. Ungedruckte Quellen
}

\author{
Frankreich
}

Archives Nationales, Paris

Série AP (Archives Privées)

Papiers Paul Reynaud (74 AP)

Papiers André Tardieu (324 AP)

Papiers Louis Marin (317 AP)

Papiers Alexandre Millerand (470 AP)

Papiers Edouard Daladier (476 AP)

Série $\mathrm{F}^{7}$ (Ministère de l'Intérieur)

12954

12955

12957

13030

13237

13254

13257

13258

13962

Série $\mathrm{F}^{60}$ (Secrétariat général du gouvernement et services du premier ministre)

474 (Textes des décrets-lois du Gouvernement Paul Reynaud, 1er avril - 5 juin 1940)

Série $2 \mathrm{~W}$ (Procès de Riom)

72 Paul Reynaud

Archives du ministère des Finances, Paris
B 21848
B 31732
B 33180
B 33190
B 33196

Archives de la Banque de France, Paris

Procès-verbaux du Conseil Général et du Comité Permanent, 1934-1945

Archives de l'Assemblée Nationale, Paris

Dossier: „Reynaud Paul“ 
Procès-verbaux des séances des Commissions et rapports annexes:

Commission des Affaires Etrangères, 1921-1924

Commission de la Réforme de l'Etat, 1934-1936

Commission de l'Armée, 1935-1936

Commission des Finances, 1930, 1934, 1938

Commission de l'Algérie, des colonies et des protectorats, 1931

Ministère des Affaires Etrangères, Paris

Série Z-Europe 1918-1940, Allemagne

Série Z-Europe 1944-1949, Généralités

Série B-Amérique, Mexique

Papiers 1940, Fonds Reynaud

Fonds Dejean

Papiers Maurice Dejean (PA-AP 288)

\section{Centre des Archives Diplomatiques de Nantes}

Botschaftsakten Berlin, Série B (1815-1939)

Botschaftsakten Mexiko, Série B (1822-1922)

Centre des Archives d'Outre-Mer, Aix-en-Provence

Série Affaires politiques

Série Indochine, Nouveau Fonds

Série Télégrammes. Cabinet du ministre des Colonies

Série Exposition coloniale internationale (1931)

Archives de Paris

Série D2M² (Elections de Paris et de l'ancien département de la Seine, 1806-1946)

Série D3M² (Propagande électorale, 1871-1961)

Bibliothèque Nationale, Paris

Papiers Alliance Républicaine Démocratique (Don 37260)

Papiers Raymond Poincaré (Nouvelles acquisitions françaises 16014)

Bibliothèque de Documentation Internationale Contemporaine, Nanterre

Dossier provisoire concernant P. Reynaud, 1940-1941

Paul Reynaud, "Je m'accuse. Mémoire pour le bâtonnier. Ecrit au Fort de Portalet“, 1. 10.

1942

Ordre des Avocats à la Cour de Paris

Dossier Paul Reynaud

Dossier „W.R.“

Discours de rentrée des conférences. Avocats stagiaires 1911-1920

Service Historique de l'Armée de Terre, Vincennes

Dossier militaire de Paul Reynaud (No 42.325/26)

Papiers Paul Reynaud (1 K 187)

Série $5 \mathrm{~N}$ (Cabinet du Ministre, 1940)

Série 7 N (Etat-Major de l'armée) 
Ecole des Hautes Etudes Commerciales, Jouy-en-Josas

Dossier Paul Reynaud

Archives Départementales des Hautes-Alpes, Gap

3 M 242-243 (Elections législatives de 1914)

Archives Départementales des Alpes-de-Haute-Provence, Digne

1 M 202 (Dossier Paul Reynaud)

1 N 42-52 (Rapports du Préfet et délibérations du Conseil Général, 1913-1940)

\section{Deutschland}

Bundesarchiv Koblenz

Nachlaß Arnold Rechberg (NL 49)

Politisches Archiv des Auswärtigen Amts, Bonn

Büro des Reichsministers

R $28189 \mathrm{k} \quad 5$ secr
R $28190 \mathrm{k}$
Büro des Staatssekretärs
R $29079 \mathrm{k} \quad$ Framil

$\mathrm{R} 28340 \mathrm{k} \quad 14-1 \mathrm{secr}$

Politische Abteilung II

R 70562

$\mathrm{R} 70563$

R 70715

$\mathrm{R} 70721$

R 70722

R 102842

R 102862

Geheimakten 1920-1936

R 30207

R 30184

\section{Reparationen (secret)}

dto.

3.21-3.23

$3.23-2.24$

Arnold Rechberg u. ähnl. illegale Aktionen zur Herbeiführung einer deutschfranzösischen Militärallianz Schritte zur Herbeiführung einer Verständigung bezügl. der Ruhraktion

Bemühungen des Arnold Rechberg u. a. zur deutschfranzösischen Verständigung dto.

Frankreich. Ministerien dto.

dto.

Eintreten des Arnold Rechberg für eine deutsch-französische Verständigung Pol. II 115. Ministerien

Frankreich. Frz. Staatsmänner "Rechberg"
$6.27-3.30$
$5.36-7.40$

1.31-8.34

2.22-6.24

$2.30-11.30$

12.30-5.31

6.36-10.39

11.29-7.31

2.23-9.32

Direktoren, Handakten (1920-36)

R 35992

Bestand Schubert 


\section{Akteneditionen, Dokumentationen, Parlamentsschrifttum}

Akten zur Deutschen Auswärtigen Politik 1918-1945, Serie A (1918-1925), Band VII, Göttingen 1989; Serie B (1925-1933), Band XI und XIII, Göttingen 1978/1979; Serie D (19371945), Band II, IV und IX, Baden-Baden/Frankfurt a.M. 1951-1962

Annales de l'Assemblée Nationale Constituante. Elue le 2 juin 1946, Débats parlementaires, Paris 1946

L'Année politique 1944/45ff., Paris $1946 \mathrm{ff}$.

Bédarida, François ( $\mathrm{Hg}$.$) , La stratégie secrète de la drôle de guerre. Le Conseil suprême in-$ terallié, septembre 1939 - avril 1940, Paris 1979

Chambre des Députés. Douzième Législature. Impressions. Projets de lois, propositions, rapports, etc. Tome XIX, No. 1431 (Programmes électoraux), Paris 1924

Chambre des Députés. Quatorzième Législature. Impressions. Projets de lois, propositions, rapports, etc. Tome XLIII, No. 3814 (Programmes électoraux), Paris 1932

Conseil de l'Europe, Assemblée Consultative. Première Session (10 août - 8 septembre 1949). Compte rendus. Band II, Strasbourg 1949

Conseil de l'Europe, Assemblée Consultative. Deuxième Session (7- 28 août 1950). Compte rendus, Strasbourg 1950

Conseil de l'Europe. Assemblée Consultative. Deuxième Session (18 - 24 novembre 1950). Comptes rendus, Band V, Strasbourg 1950

Council of Europe, Consultative Assembly. First Session (10th August - 8th September 1949), Agendas. Minutes, Strasbourg 1949

Council of Europe, Consultative Assembly. Second Session 1950. Report of the Committee on Economic Questions, Strasbourg 1950

Département de la Seine. Elections à la Chambre des Députés. Mai 1924-Mars 1928. Résultats par Arrondissements pour les circonscriptions de Paris et par communes pour la circonscription des arrondissements de Saint-Denis et de Sceaux, Paris 1928

Documents Diplomatiques Français 1932-1939; 1re série (1932-1935), Band II, Paris 1964; 2e série (1936-1939), Band XIII, Paris 1973

Documents on British Foreign Policy 1919-1939, First Series, Band VIII, London 1958; Third Series, Band I und II, London 1949

Exposition Coloniale Internationale 1931, Paris. Rapport général présenté par le gouverneur général Olivier, Rapporteur général, délégué général à l'Exposition. 7 Bände, Paris 1932-34

Les événements survenus en France 1933-1945. Témoignages et documents recueillis par la commission d'enquête parlementaire, Band 1-9, Paris 1947-1951

Foreign Relations of the United States. Diplomatic Papers 1940, Band I, Washington 1959

Foreign Relations of the United States 1948. Band II: Germany and Austria, Washington 1973

Foreign Relations of the United States 1948. Band III: Western Europe, Washington 1974

Foreign Relations of the United States 1950. Band III: Western Europe, Washington 1977

For the President. Personal and Secret Correspondance between F.D. Roosevelt and W.C. Bullitt, Boston 1972

Die Geheimakten des französischen Generalstabes. Weißbuch des Auswärtigen Amtes, Band 6, Berlin 1941

Gilbert, Martin (Hg.), The Churchill War Papers. Band I: At the Admiralty (September 1939 - May 1940), London 1993

Journal Officiel, Assemblée Nationale, Débats parlementaires, Paris $1947 \mathrm{ff}$.

Journal Officiel, Chambre des Députés, Débats parlementaires, Paris 1919-1940

Journal Officiel, Chambre des Députés, Débats parlementaires, Comités secrets, Paris 1948

Die Kabinette Wirth I und II. 10. Mai 1921 bis 26. Oktober 1921. 26. Oktober 1921 bis 22. November 1922. Band I, bearb. von Ingrid Schulze-Bidlingmaier, Boppard 1973

Lipgens, Walter/Loth, Wilfried (Hg.), Documents on the History of European Integration. Band III: The Struggle for European Union by Political Parties and Pressure Groups in Western European Countries 1945-1950, Berlin/New York 1988 
Das Londoner Deutschland-Kommuniqué vom 7. Juni 1948, in: Europa-Archiv 3 (1948), S. 1437-1439

Le procès du Maréchal Pétain. Compte rendu sténographique, Paris 1945

\section{Memoiren, Tagebücher, Gesprächsaufzeichnungen, Schriften Reynauds}

Adenauer, Konrad, Teegespräche 1950-1954 (Adenauer. Rhöndorfer Ausgabe), Berlin 1984 Ders., Teegespräche 1959-1961 (Adenauer. Rhöndorfer Ausgabe), Berlin 1988

Aron, Raymond, Mémoires. 50 ans de réflexion politique, Paris 1983

Auriol, Vincent, Journal du Septennat 1947-1954. 7 Bände, Paris 1970-78

Bardoux, Jacques, Journal d'un témoin de la Troisième, 1er septembre 1939 - 15 juillet 1940, Paris 1957

Baudouin, Paul, Neuf mois au gouvernement (avril - décembre 1940), Paris 1948

The private diaries of Paul Baudouin (March 1940 to January 1941), London 1948

Beneš, Edvard, Der Aufstand der Nationen. Der Weltkrieg und die tschechoslowakische Revolution, Prag 1928

Bloch, Marc, L'étrange défaite. Témoignage écrit en 1940, Paris 1990

L'Oeuvre de Léon Blum. Band III/1 (1914-1928), Paris 1972; Band III,2 (1928-1934), Paris 1972; Band V: Mémoires. Le prison et le procès. A l'échelle humaine (1940-1945), Paris 1955

Bonnet, Georges, Quai d'Orsay, Isle of Man 1965

Charles-Roux, François, Cinq mois tragiques aux affaires étrangères (21 mai - 1er novembre 1940), Paris 1949

Chautemps, Camille, Cahiers secrets de l'armistice (1939-1940), Paris 1963

Chauvel, Jean, Commentaires. Band I: De Vienne à Alger (1938-1944), Paris 1971

Churchill, Winston S., The Second World War. Band I: The Gathering Storm, London 1948; Band II: Their Finest Hour, Boston 1949

Dalton, Hugh, High Tide and After. Memoirs 1945-1960, London 1962

Debré, Michel, Trois Républiques pour une France. Mémoires. Band I: Combattre, Paris 1984

Duclos, Jacques, Mémoires. Band I: 1896-1934. Le chemin que j’ai choisi. De Verdun au Parti communiste, Paris 1968

Fabre-Luce, Alfred, Journal de la France, mars 1939-juillet 1940, Paris 1940

Fabry, Jean, J'ai connu. 2 Bände, Paris 1960

Flandin, Pierre-Etienne, Paix et Liberté. L'Alliance démocratique à l'action, Paris 1938

Gamelin, Maurice, Servir. Band III, Paris 1947

Gaulle, Charles de, Lettres, notes et carnets. 1919-juin 1940, Paris 1980

Ders., Lettres, notes et carnets. Juin 1940 - juillet 1941, Paris 1981

Ders., Lettres, notes et carnets. Juillet 1941 - mai 1943, Paris 1982

Ders., Mémoires de Guerre. Band I: L'Appel 1940-42, Paris 1954

Harvey, John (Hg.), The Diplomatic Diaries of Oliver Harvey 1937-1940, New York 1970

Jeanneney, Jules, Journal politique. Septembre 1939 - Juillet 1942. Edition établie, présentée et annotée par Jean-Noël Jeanneney, Paris 1972

Laniel, Joseph, Jours de gloire et jours cruels 1908-1958, Paris 1971

Lazareff, Pierre, De Munich à Vichy, New York 1944

Leca, Dominique, La rupture de 1940, Paris 1978

Marbo, Camille, A travers deux siècles. Souvenirs et rencontres (1883-1967), Paris 1968

Marjolin, Robert, Le travail d'une vie. Mémoires 1911-1986, Paris 1986

Massigli, René, Une comédie des erreurs 1943-1956. Souvenirs et réflexions sur une étape de la construction européenne, Paris 1978 
Monnet, Jean, Mémoires, Paris 1976

Monzie, Anatole de, Ci-devant, Paris 1941

Palewski, Gaston, Mémoires d'action, 1924-1974, Paris 1988

Poincaré, Raymond, Histoire politique. Chroniques de quinzaine, 4 Bände, Paris 1920-22

Pomaret, Charles, Le dernier témoin. Fin d'une guerre, fin d'une république. Juin et juillet 1940, Paris 1968

Popelin, Claude, Arènes politiques, Paris 1974

Reynaud, Paul, Des Concordats de Sociétés anonymes après faillite ou liquidation judiciaire, Paris 1904

Ders., Waldeck-Rousseau, Paris 1913

Ders., Les Trois Glorieuses, Paris 1927

Ders., Jeunesse, quelle France veux-tu?, Paris 1936

Ders., Le problème militaire français, Paris 1937

Ders., Courage de la France, Paris 1939

Ders., Finances de guerre, Paris 1940

Ders., Le destin hésite, Paris 1946

Ders., La France a sauvé l'Europe. 2 Bände, Paris 1947

Ders., Au coeur de la mêlée, Paris 1951

Ders., S'unir ou périr, Paris 1951

Ders., Mémoires. Band I: Venu de ma montagne, Paris 1960; Band II: Envers et contre tous, Paris 1963

Ders., La politique étrangère du Gaullisme, Paris 1964 [dt.: Ehrgeiz und Illusion. Die Außenpolitik de Gaulles, München/Zürich 1964]

Ders., Et après?, Paris 1964

Ders., Carnets de captivité 1941-1945. Présentés par Evelyne Demey, Paris 1997

Sartre, Jean-Paul, Les carnets de la drôle de guerre. Novembre 1939-Mars 1940, Paris 1983

Spears, Edward, Assignment to Catastrophe. Band II: The Fall of France, June 1940, Melbourne u. a. 1954

Trefusis, Violet, Prélude au désastre, Paris 1990

Villelume, Paul de, Journal d'une défaite. (23 Août 1939 - 16 Juin 1940), Paris 1976

Zay, Jean, Carnets secrets (De Munich à la guerre), Paris 1942

\section{Presse}

L'Action Française

L'Agence Economique et Financière

L'Alliance Démocratique

L'Alliance Républicaine Démocratique

Les Alpes Républicaines

L'Ami du Peuple

L'Aube

L'Avenir

Le Capital

Ce Soir

Le Cri de Paris

Le Charivari

L'Echo de Paris

L'Eclair

L'Ere Nouvelle

Esprit

L'Excelsior

Le Figaro 
France-Soir

Gringoire

L'Humanité

L'Information

L'Intransigeant

Le Journal

Le Journal de Barcelonnette

Le Journal de la Bourse

Le Journal des Débats

La Journée Industrielle

La Liberté

Le Matin

Le Monde

La Nation

Les Nouvelles économiques et financières

L'Oeuvre

L'Ordre

Paris-Midi

Paris-Soir

La Patrie

Le Petit Journal

Le Petit Parisien

Le Populaire

Le Radical

La Renaissance

Le Républicain National du 2e Arrondissement

La République

La République Démocratique

Le Réveil du Deuxième

La Revue Hebdomadaire

Sept-Jours

La Situation économique et financière

Le Temps

La Victoire

La Volonté

\section{Darstellungen}

Adamthwaite, Antony, France and the Coming of the Second World War 1936-1939, London 1977

Ders., Grandeur and misery. France's bid for power in Europe 1914-1940, London u. a. 1995

Ageron, Charles-Robert, France coloniale ou parti colonial?, Paris 1978

Ders., L'exposition coloniale de 1931. Mythe républicain ou mythe impérial?, in: Pierre Nora (Hg.), Les Lieux de mémoire. Band I: La République, Paris 1984, S. 561-591

Albertini, Rudolf von, Die Diskussion um die französische Steuerreform, 1907-1909, in: Schweizer Beiträge zur allgemeinen Geschichte 13 (1955), S. 183-201

Ders., Regierung und Parlament in der Dritten Republik, in: HZ 188 (1959), S. 17-48

Ders., Parteiorganisation und Parteibegriff in Frankreich, in: HZ 193 (1961), S. 529-600

Ders., Dekolonisation. Die Diskussion über Verwaltung und Zukunft der Kolonien 19191960, Köln/Opladen 1966 
Alexander, Martin S., The Republic in danger. General Maurice Gamelin and the politics of French defence, 1933-1940, Cambridge 1992

Alter, Peter, Nationalismus, Frankfurt a. M. 1985

Anderson, Malcolm, Conservative Politics in France, London 1974

Antiq-Auvaro, Raymonde, L'émigration des Barcelonnettes au Mexique, Nice 1992

Arnaud, François u.a., Les Barcelonnettes au Mexique. Récits et témoignages, Barcelonnette 31994

Aron, Raymond/Lerner, Daniel (Hg.), La querelle de la C.E.D. Essais d'analyse sociologique, Paris 1956

Aron, Robert, Histoire de l'Epuration. Band II: Des prisons clandestines aux tribunaux d'exception, septembre 1944 - juin 1949, Paris 1969

Artaud, Denise, A propos de l'occupation de la Ruhr, in: Revue de l'Histoire Moderne et Contemporaine 17 (1970), S. 1-21

Dies., La question des dettes interalliées et la reconstruction de l'Europe (1917-1929). 2 Bände, Paris 1978

Dies., Die Hintergründe der Ruhrbesetzung 1923. Das Problem der interalliierten Schulden, in: VfZ 27 (1979), S. 241-259

Dies., La question des dettes interalliées et la reconstruction de l'Europe, in: Revue Historique 261 (1979), S. 363-382

Asselain, Jean-Charles, Une erreur de politique économique: la loi des quarante heures de 1936, in: Revue économique 25/4 (1974), S. 672-705

Ders., Histoire économique de la France du XVIIIe siècle à nos jours. Band II: De 1919 à la fin des années 1970, Paris 1984

Aubert, Louis/Martin, Ivan/Missoffe, Michel/Piétri, François/Pose, Alfred, André Tardieu, Paris 1957

Auburtin, Jean, Le Colonel de Gaulle, Paris 1965

Audigier, François, L'Alliance démocratique de 1933 à 1937 ou l'anachronisme en politique, in: Vingtième Siècle 47 (1995), S. 147-157

Auerbach, Hellmuth, Die europäische Wende der französischen Deutschlandpolitik 1947/ 48, in: Ludolf Herbst/Werner Bührer/Hanno Sowade (Hg.), Vom Marshallplan zur EWG. Die Eingliederung der Bundesrepublik Deutschland in die westliche Welt, München 1990; S. 577-591

Avril, Pierre, Droit de dissolution, in: Jean-François Sirinelli (Hg.), Dictionnaire historique de la vie politique française au XXe siècle, Paris 1995, S. 308-311

Azéma, Jean-Pierre, De Munich à la Libération, 1938-1944 (Nouvelle Histoire de la France Contemporaine 14), Paris 1979

Ders., Die französische Politik am Vorabend des Krieges, in: Wolfgang Benz/Hermann Graml (Hg.), Sommer 1939. Die Großmächte und der Europäische Krieg, Stuttgart 1979, S. 280-313

Ders., 1940. L'année terrible, Paris 1990

Ders./Winock, Michel, La troisième République (1870-1940), Paris 21976

Baal, Gérard, Le radicalisme, Paris 1994

Ballensiefen, Heinz, Frankreichs Schuld. Die inneren Gründe für den Zusammenbruch des französischen Staates, in: Zeitschrift für Politik 6/7 (1940), S. 261-282

Bankwitz, Philip C.F., Maxime Weygand and the Fall of France: A Study in Civil-Military Relations, in: The Journal of Modern History 31 (1959), S. 225-242

Ders., Maxime Weygand and civil-military relations in modern France, Cambridge/Mass. 1967

Bariéty, Jacques, Les relations franco-allemandes après la Première Guerre Mondiale. 10 Novembre 1918-10 janvier 1925. De l'Exécution à la Négociation, Paris 1977

Ders., Un artisan méconnu des relations franco-allemandes: le professeur Oswald Hesnard, 1877-1936, in: Media in Francia... Recueil de mélanges offert à Karl Ferdinand Werner à l'occasion de son $65^{\mathrm{e}}$ anniversaire par ses amis et collègues français, Paris 1989, S. 1-18

Ders., Frankreich und das deutsche Problem nach dem Ersten und nach dem Zweiten Welt- 
krieg, in: Klaus Schwabe/Francesca Schinzinger (Hg.), Deutschland und der Westen im 19. und 20. Jahrhundert. Teil 2: Deutschland und Westeuropa, Stuttgart 1994, S. 121-136

Barker, Elisabeth, British Policy in South-East Europe in the Second World War, London 1976

Bartel, Heinrich, Frankreich und die Sowjetunion 1938-1940. Ein Beitrag zur französischen Ostpolitik zwischen dem Münchener Abkommen und dem Ende der Dritten Republik, Stuttgart 1986

Basso, Jacques, Les élections législatives dans le département des Alpes-Maritimes 18601939, Paris 1968

Beau de Loménie, Emanuel, La mort de la Troisième République, Paris 1951

Beaufre, André, Le drame de 1940, Paris 1965

Becker, Jean-Jacques, 1914: Comment les Français sont entrés dans la guerre. Contribution à l'étude de l'opinion publique, printemps-été 1914, Paris 1977

Ders./Berstein, Serge, Victoire et frustrations 1914-1929 (Nouvelle histoire de la France contemporaine 12), Paris 1990

Bédarida, François, La "gouvernante anglaise“, in: Rémond/Bourdin (Hg.), Edouard Daladier, S. 228-240

Ders., Convergences et divergences stratégiques franco-britanniques, in: Français et Britanniques dans la Drôle de guerre. Actes du colloque franco-britannique tenu à Paris du 8 au 12 décembre 1975, Paris 1979, S. 359-378

Ders., La rupture franco-britannique de 1940. Le Conseil Suprême Interallié, de l'invasion à la défaite de la France, in: Vingtième Siècle 25 (1990), S. 37-48

Bell, David S./Johnson, Douglas/Morris, Peter (Hg.), Biographical Dictionary of French Political Leaders since 1870, New York u. a. 1990

Bellstedt, Hans F., „Apaisement“ oder Krieg. Frankreichs Außenminister Georges Bonnet und die deutsch-französische Erklärung vom 6. Dezember 1938 (Pariser Historische Studien 37), Bonn 1993

Bendikat, Elfi, Wahlkämpfe in Europa 1884-1889. Parteiensysteme und Politikstile in Deutschland, Frankreich und Großbritannien, Wiesbaden 1988

Benoist, Charles, Le suffrage universel et l'évolution des partis politiques, in: Revue des Deux Mondes 2 (1904), S. $520 \mathrm{ff}$.

Ders., Comment on capte le suffrage et le pouvoir, in: Revue des Deux Mondes 3 (1904), S. $885 \mathrm{ff}$.

Benz, Wolfgang/Graml, Hermann (Hg.), Biographisches Lexikon zur Weimarer Republik, München 1988

Berghahn, Volker R., Der Stahlhelm. Bund der Frontsoldaten 1918-1935 (Beiträge zur Geschichte des Parlamentarismus und der politischen Parteien 33), Düsseldorf 1966

Berl, Emmanuel, La fin de la IIIe République. 10 juillet 1940, Paris 1968

Berstein, Serge, Le 6 février 1934, Paris 1975

Ders., Les conceptions du Parti radical en matière de politique économique extérieure, in: Relations internationales 13 (1978), S. 71-89

Ders., Le parti radical-socialiste arbitre du jeu politique français, in: René Rémond/Janine Bourdin (Hg.), La France et les Français en 1938/1939, Paris 1978, S. 275-306

Ders., Histoire du Parti Radical. 2 Bände, Paris 1980/1982

Ders., Les partis, in: René Rémond (Hg.), Pour une histoire politique, Paris 1988, S. 49-85

Ders., La politique sociale des Républicains, in: Ders./Odile Rudelle (Hg.), Le modèle républicain, Paris 1992, S. 189-208

Berstein, Gisèle et Serge, La Troisième République. Les noms, les thèmes, les lieux, Paris 1987

Berstein, Serge/Becker, Jean-Jacques, Histoire de l'anticommunisme en France. Band I: 1917-1940, Paris 1987

Berstein, Serge/Milza, Pierre, Histoire de la France au XXe siècle. Band I: 1900-1930, Brüssel 1990; Band II: 1930-1945, Paris 1991

Berstein, Serge/Rudelle, Odile (Hg.), Le modèle républicain, Paris 1992 
Betts, Raymond F., Assimilation and Association in French Colonial Theory 1890-1914, New York/London 1961

Beyme, Klaus von, Die parlamentarischen Regierungssysteme in Europa, München 21973

Binion, Rudolph, Defeated Leaders. The political fate of Caillaux, Jouvenel and Tardieu, New York 1960

Birke, Ernst, Der Erste Weltkrieg und die Gründung der Tschechoslowakei 1914-1919, in: Karl Bosl (Hg.), Handbuch der Geschichte der böhmischen Länder. Band III, Stuttgart 1968, S. 239-446

Birnbaum, Pierre, Les sommets de l'Etat. Essai sur l'élite du pouvoir en France, Paris 1977

Blatt, Joel (Hg.), The French Defeat of 1940. Reassessments, Providence/Oxford 1998

Blet, Henri, France d'outre-mer: l'oeuvre coloniale de la IIIe République, Grenoble 1950

Bloch, Charles, Die Dritte Französische Republik. Entwicklung und Kampf einer parlamentarischen Demokratie (1870-1940), Stuttgart 1972

Bock, Hans Manfred, „Connaître l'Allemagne et la reconnaître.“ Zu Entstehung und Zusammenhang der Deutschland-Analyse von Pierre Viénot 1922 bis 1932, in: Lendemains 66 (1992), S. 27-48

Ders., Friedrich Sieburg et Pierre Viénot ou la fin du „Locarno intellectuel“, in: Allemagnes d'aujourd'hui 105 (1988), S. 84-99

Ders., Zur Perzeption der frühen Bundesrepublik Deutschland in der französischen Diplomatie: Die Bonner Monatsberichte des Hochkommissars André François-Poncet 19491955, in: Francia 15 (1987), S. 579-658

Böhme, Hermann, Der deutsch-französische Waffenstillstand im Zweiten Weltkrieg, Band I: Entstehung und Grundlagen des Waffenstillstandes von 1940 (Quellen und Darstellungen zur Zeitgeschichte 12), Stuttgart 1966

Boldt, Hans, Deutsche Verfassungsgeschichte. Politische Strukturen und ihr Wandel. Band II: Von 1806 bis zur Gegenwart, München 21993

Boll, Friedhelm, Arbeitskämpfe und Gewerkschaften in Deutschland, England und Frankreich. Ihre Entwicklung vom 19. zum 20. Jahrhundert, Bonn 1992

Bond, Brian, Britain, France and Belgium 1939-1940, London u.a. 21990

Bonnard, Abel, Les modérés. Le drame du présent, Paris 1936

Bonnefous, Georges, Histoire politique de la Troisième République. 7 Bände, Paris 19591984

Borne, Dominique/Dubief, Henri, La crise des années 30 (1929-1938), (Nouvelle histoire de la France contemporaine 13), Paris 21989

Bossuat, Gérard, La France, l'aide américaine et la construction européenne 1944-1954. 2 Bände, Paris 1992

Ders., Le poids de l'aide américaine sur la politique économique et financière de la France en 1948, in: Relations internationales 37 (1984), S. 17-36

Boulic, Jean-Yves/Lavaure, Anne, Henri de Kerillis 1889-1958. L'absolu patriote, Rennes 1997

Bourdrel, Philippe, La Cagoule. Histoire d'une société secrète du Front populaire à la Ve République, Paris 1992

Bourgin, Georges/Carrère, Jean/Guérin, André, Manuel des partis politiques en France, Paris $1924,{ }^{2} 1928$

Bracher, Karl Dietrich, Europa in der Krise. Innengeschichte und Weltpolitik seit 1917, Frankfurt a. M. u. a. 1979

Braudel, Fernand/Labrousse, Ernest (Hg.), Histoire économique et sociale de la France. Band IV: L'ère industrielle et la société d'aujourd'hui (siècle 1880-1980). 3 Teilbände, Paris 1979-1982

Braunias, Karl, Staatskrise und Staatsreform in Frankreich, in: Jahrbuch des Öffentlichen Rechts der Gegenwart 23 (1936), S. 72-135

Brender, Reinhold, Kollaboration in Frankreich im Zweiten Weltkrieg. Marcel Déat und das Rassemblement national populaire, München 1992

Brocheux, Pierre/Hémery, Daniel, Indochine. La colonisation ambiguë (1858-1954), Paris 1995 
Broustra, Jean-Claude, Le combat de Raymond Patenôtre, Paris 1969

Brugmans, Hendrik, Der Haager Kongreß nach vierzig Jahren - Reflexionen eines Zeitzeugen, in: Integration 11 (1988), S. 47-55

Bruhat, Jean, La CGT, in: René Rémond/Janine Bourdin (Hg.), La France et les Français en 1938/1939, Paris 1978, S. 159-188

Brun, Gérard, Technocrates et technocratie en France, 1918-1945, Paris 1985

Brunet, Jean-Paul, La police de l'ombre. Indicateurs et provocateurs dans la France contemporaine, Paris 1990

Brunschwig, Henri, Mythes et réalités de l'impérialisme colonial français, 1871-1914, Paris 1960

Bührer, Werner, Rechberg, Arnold, in: Wolfgang Benz/Hermann Graml (Hg.), Biographisches Lexikon zur Weimarer Republik, München 1988, S. $265 \mathrm{f}$.

Burdeau, Georges, Le libéralisme, Paris 1979

Ders./Hamon, Francis/Troper, Michel, Droit constitutionnel, Paris 1993

Butterworth, Suzan B., Daladier and the Munich Crisis. A Reappraisal, in: Journal of Contemporary History 9/3 (1974), S. 191-216

Capitant, René, La crise et la réforme du Parlementarisme en France. Chronique constitutionnelle française (1931-1936), in: Jahrbuch des Öffentlichen Rechts der Gegenwart 23 (1936), S. 1-71

Carley, Michael Jabora, Revolution and intervention: the French Government and the Russian civil war, 1917-1919, Kingston 1983

Carlier, Claude/Martens, Stefan (Hg.), La France et l'Allemagne en guerre. Septembre 1939Novembre 1942, Paris 1990

Carls, Stephen D., Louis Loucheur and the Shaping of Modern France 1916-1931, Baton Rouge/London 1993

Caron, François, Histoire économique de la France, XIXe-XXe siècles, Paris 1981

Carré, J.-J./Dubois, P./Malinvaud, E., French Economic Growth, Stanford 1975

Castellan, Georges, Le réarmement clandestin de l'Allemagne dans l'entre-deux-guerres, in: Revue d'Allemagne 8 (1976), S. 61-82

Ders., Le réarmement clandestin du Reich, 1930-1935. Vu par le 2e Bureau de l'Etat-Major français, Paris 1954

Cavaillé, Marie-Dominique, Rudolf Breitscheid et la France, 1919-1933, Frankfurt a.M. u.a. 1995

Chapsal, Jacques, La vie politique en France de 1940 à 1958, Paris 1984

Chevallier, Jean-Jacques, Histoire des institutions et des régimes politiques de la France de 1789 à nos jours, Paris 71985

Clague, Monique, Vision and Myopia in the New Politics of Andre Tardieu, in: French Historical Studies 8 (1973/74), S. 105-129

Cole, Alistair/Campbell, Peter, French Electoral Systems and Elections since 1789, Aldershot u. a. 1989

Connors, Joseph D., Paul Reynaud and French National Defense, 1933-1939, Diss. Chicago 1977

Conquet, Alfred, Auprès du Maréchal Pétain, Paris 1970

Coquery-Vidrovitch, Catherine, La colonisation française 1931-1939, in: Jacques Thobie/ Gilbert Meynier/Catherine Coquery-Vidrovitch/Charles-Robert Ageron, Histoire de la France coloniale. Band II: 1914-1990, Paris 1990, S. 211-308

Dies., L'Afrique coloniale française et la crise de 1930: crise structurelle et genèse du sousdéveloppement, in: Revue française d'histoire d'outre-mer 63 (1976), S. 386-424

Cornides, Wilhelm, Der Europarat und die nationalen Probleme, in: Europa-Archiv 6 (1951), S. 3655-3676

Coutau-Bégarie, Hervé/Huan, Claude, Darlan, Paris 1989

Crémieux-Brilhac, Jean-Louis, Les Français de l'an 40. 2 Bände, Paris 1990

Ders., L'opinion publique française, l'Angleterre et la guerre (septembre 1939 - juin 1940), in: Français et Britanniques dans la Drôle de guerre, S. 1-50 
De la Gorce, Paul-Marie, L'après-guerre 1944-1952. Naissance de la France moderne, Paris 1978

Delperrié de Bayac, Jacques, Histoire du Front populaire, Paris 1972

Demey, Evelyne, Paul Reynaud, mon père, Paris 1980

Dendias, Michel, Le renforcement des pouvoirs du chef de l'état dans la démocratie parlementaire, Paris 1932

Didier, Camille, L'accord de Wiesbaden au point de vue de l'industrie et des sinistrés français, Paris 1921

Dogan, Mattei, Les filières de la carrière politique en France, in: Revue française de Sociologie 8 (1967), S. 468-492

Dohrmann, Bernd, Die englische Europapolitik in der Wirtschaftskrise 1921-1923. Zur Interdependenz von Wirtschaftsinteressen und Außenpolitik, München u.a. 1980

Doughty, Robert Allan, The Seeds of Disaster. The Development of French Army Doctrine 1919-1939, Hamden/Conn. 1985

Duguit, Léon, Traité de droit constitutionnel. Band I, Paris 1921

Duhamel, Olivier, Histoire constitutionnelle de la France, Paris 1995

Duroselle, Jean-Baptiste, La décadence 1932-1939, Paris 1979

Ders., L'abîme 1939-1944, Paris 21986

Dutailly, Henry, Les problèmes de l'armée de terre française (1935-1939), Paris 1980

Ders., Programmes d'armement et structures modernes dans l'armée de terre (1935-1939), in: Klaus Hildebrand/Karl Ferdinand Werner (Hg.), Deutschland und Frankreich 1936-1939. 15. Deutsch-französisches Historikerkolloquium des Deutschen Historischen Instituts Paris (Bonn, 26.-29. September 1979), München 1981, S. 105-128

Duverger, Maurice, Le système politique français. Droit constitutionnel et systèmes politiques, Paris ${ }^{18} 1985$

Eck, Jean-François, Louis Marin, in: Jean-François Sirinelli (Hg.), Dictionnaire historique de la vie politique française au XXe siècle, Paris 1995, S. $630 \mathrm{f}$.

Ehrmann, Henry, The Blum Experiment and the Fall of France, in: Foreign Affairs 10 (1941), S. $152-164$

Eichengreen, Barry/Sachs, Jeffrey, Exchange Rates and Economic Recovery in the 1930s, in: Journal of Economic History 45 (1985), S. 925-946

Elwitt, Sanford, The Third Republic Defended. Bourgeois Reform in France, 1880-1914, Baton Rouge/London 1986

Esmein, Adhémar, Eléments du droit constitutionnel français et comparé. Band II: Le droit constitutionnel de la République française, Paris ${ }^{4} 1906,{ }^{5} 1909,81928$

Ewald, François, La politique sociale des opportunistes, 1879-1885, in: Berstein/Rudelle (Hg.), Modèle républicain, S. 173-187

Farrar, Majorie M., Principled Pragmatist. The Political Career of Alexandre Millerand, New York/Oxford 1991

Feldman, Gerald D., The Great Disorder. Politics, Economics, and Society in the German Inflation, 1914-1924, New York u. a. 1993

Feldman, Gerald D./Homburg, Heidrun, Industrie und Inflation. Studien und Dokumente zur Politik der deutschen Unternehmer 1916-1923, Hamburg 1977

Feldman, Gerald D. (Hg.), Die Nachwirkungen der Inflation auf die deutsche Geschichte 1924-1933, München 1985

Ferro, Marc, Der große Krieg. 1914-1918, Frankfurt 1988

Finer, Herman, The Theory and Practice of modern Government. Band I, New York 1946

Français et Britanniques dans la Drôle de guerre. Actes du colloque franco-britannique tenu à Paris du 8 au 12 décembre 1975, Paris 1979

Fraenkel, Ernst, Die repräsentative und plebiszitäre Komponente im demokratischen Verfassungsstaat, in: Ders., Deutschland und die westlichen Demokratien. Erweiterte Ausgabe, Frankfurt a.M. 1991, S. 153-203

Frankenstein, Robert, Le prix du réarmement français (1935-1939), Paris 1982 
Ders., Le financement français de la guerre et les accords avec les Britanniques (1939-1940), in: Français et Britanniques dans la Drôle de guerre, S. 461-487

Frayssinet, Jean, Le Conseil économique et social, Paris 1986

Frieser, Karl-Heinz, Blitzkrieg-Legende. Der Westfeldzug 1940 (Operationen des Zweiten Weltkrieges 2), München 1995

Fritsch-Bournazel, Renata, Rapallo: naissance d'un mythe. La politique de la peur dans la France du Bloc national, Paris 1974

Furet, François, Penser la Révolution française, Paris 1978

Gall, Lothar (Hg.), Liberalismus, Königstein/Ts. ${ }^{3} 1985$

Ders., Einleitung, in: Ders. (Hg.), Liberalismus, Königstein/Ts. ${ }^{31985, ~ S . ~ 9-19 ~}$

Gates, Eleanor M., End of the Affair. The Collapse of the Anglo-French Alliance, 1939-40, Berkeley/Los Angeles 1981

Gaudry, Olivier, Henri de Kerillis, Mémoire IEP Paris 1966

Gicquel, Jean, Droit constitutionnel et institutions politiques, Paris ${ }^{10} 1989$

Gicquel, Jean/Sfez, Lucien, Problèmes de la réforme de l'état en France depuis 1934, Paris 1965

Gilbert, Martin, Winston S. Churchill. Band V (1922-1939), London 1976

Gimbel, John, The origins of the Marshall Plan, Stanford 1976

Girard, Louis, Les libéraux français 1814-1875, Paris 1985

Girardet, Raoul, L'apothéose de la „plus grande France“. L'idée coloniale devant l'opinion française (1930-1935), in: Revue française de science politique 18 (1968), S. 1085-1114

Ders., L'idée coloniale en France de 1871-1962, Paris 1972

Girault, René, Les relations franco-soviétiques après septembre 1939, in: Français et Britanniques dans la Drôle de guerre, S. 263-279

Ders., La décision gouvernementale en politique extérieure, in: Rémond/Bourdin (Hg.), Edouard Daladier, S. 209-227

Ders., La politique extérieure française de l'après-Munich, septembre 1938 - avril 1939, in: Hildebrand/Werner, Deutschland und Frankreich 1936-1939, S. 507-522

Ders., Les décideurs français et la puissance française en 1938/1939, in: Ders./Robert Frank, Histoire des Relations Internationales, Band II: Turbulente Europe et nouveaux mondes (1914-1941), Paris 1988, S. 23-43

Glum, Friedrich, Der deutsche und der französische Reichswirtschaftsrat. Ein Beitrag zu dem Problem der Repräsentation der Wirtschaft im Staat, Berlin 1929

Goguel, François, La politique des partis sous la IIIe République, Paris 31953

Ders., Les élections législatives et sénatoriales partielles, in: Rémond/Bourdin ( $\mathrm{Hg}$.), Edouard Daladier, S. 45-54

Ders., L'incapacité de la IIIe République à réformer ses institutions, in: Paul Isoart/Christian Bidegaray (Hg.), Des Républiques françaises, Paris 1988, S. 664-673

Graham, Bruce D., Choice and Democratic Order. The French Socalist Party, 1937-1950, Cambridge 1994

Greilsammer, Ilan, Blum, Paris 1996

Grèzes-Rueff, François, La culture des députés français (1910-1958). Essai de typologie, Toulouse 1994

Griffiths, Richard, Hervé, Gustave, in: Biographical Dictionary of French Political Leaders since 1870, New York u.a. 1990, S. $203 \mathrm{f}$.

Grosser, Alfred, La Quatrième République et sa politique extérieure, Paris 31972

Ders., Frankreich und seine Außenpolitik 1944 bis heute, München/Wien 1986

Grüner, Stefan, Vom Umgang mit der demokratischen Vergangenheit. Das Deutsche Reich, Vichy und das politische Führungspersonal der Dritten Französischen Republik 19401945, in: Stefan Martens/Maurice Vaïsse (Hg.), Frankreich und Deutschland im Krieg (November 1942-Herbst 1944). Okkupation, Kollaboration, Résistance, Paris 2000, S. 73-98

Guillen, Pierre, Die französische Generalität, die Aufrüstung der Bundesrepublik und die EVG (1950-1954), in: Hans-Erich Volkmann/Walter Schwengler (Hg.), Die Europäische 
Verteidigungsgemeinschaft. Stand und Probleme der Forschung (Militärgeschichte seit 1945, Band 7), Boppard a. Rh. 1985

Guiral, Pierre, Der Liberalismus in Frankreich (1815-1870), in: Lothar Gall (Hg.), Liberalismus, Königstein/Ts. ${ }^{31985}$, S. 283-307

Hagspiel, Hermann, Verständigung zwischen Deutschland und Frankreich? Die deutschfranzösische Außenpolitik der zwanziger Jahre im innenpolitischen Kräftefeld beider Länder (Pariser Historische Studien 24), Bonn 1987

Hatzfeld, Henri, Du paupérisme à la sécurité sociale 1850-1940. Essai sur les origines de la Sécurité sociale en France, Nancy 1989

Haupt, Heinz-Gerhard, Sozialgeschichte Frankreichs seit 1789, Frankfurt a. M. 1989

Heimsoeth, Hans-Jürgen, Der Zusammenbruch der Dritten Französischen Republik. Frankreich während der „Drôle de Guerre“ 1939/1940 (Pariser Historische Studien 30), Bonn 1990

Ders., Frankreich im Krieg mit Deutschland: Der Zerfall der Dritten Französischen Republik während der „Drôle de guerre“, in: Claude Carlier/Stefan Martens (Hg.), La France et l'Allemagne en guerre. Septembre 1939 - Novembre 1942, Paris 1990, S. 49-67

Hémery, Daniel, Révolutionnaires vietnamiens et pouvoir colonial en Indochine. Communistes, trotskystes, nationalistes à Saigon de 1932 à 1937, Paris 1975

Ders., Aux origines des guerres d'indépendance vietnamiennes: pouvoir colonial et phénomène communiste en Indochine avant la Seconde Guerre mondiale, in: Le Mouvement social 101 (1977), S. 3-35

Herbst, Ludolf/Bührer, Werner/Sowade, Hanno (Hg.), Vom Marshallplan zur EWG. Die Eingliederung der Bundesrepublik Deutschland in die westliche Welt (Quellen und Darstellungen zur Zeitgeschichte 30), München 1990

Heyde, Philipp, Das Ende der Reparationen. Deutschland, Frankreich und der Youngplan 1929-1932, Paderborn u. a. 1998

Hilaire, Yves-Marie, 1900-1945. L'ancrage des idéologies, in: Jean-François Sirinelli (Hg.), Histoire des droites en France. Band I, Paris 1992, S. 519-565

Hildebrand, Klaus, Krieg im Frieden und Frieden im Krieg. Über das Problem der Legitimität in der Geschichte der Staatengesellschaft 1931-1941, in: HZ 244 (1987), S. 1-28

Ders., Das vergangene Reich. Deutsche Außenpolitik von Bismarck bis Hitler 1871-1945, Stuttgart 1995

Hildebrand, Klaus/Werner, Karl Ferdinand (Hg.), Deutschland und Frankreich 1936-1939. 15. Deutsch-französisches Historikerkolloquium des Deutschen Historischen Instituts Paris (Bonn, 26.-29. September 1979), München 1981

Hirsch, Julius, Die deutsche Währungsfrage (Kieler Vorträge 9), Jena 1924

Höhne, Roland/Kolboom, Ingo, Sozialliberalismus in Frankreich. Ambivalenzen und Grenzen des französischen Liberalismus in Geschichte und Gegenwart, in: Karl Holl/Günter Trautmann/Hans Vorländer (Hg.), Sozialer Liberalismus, Göttingen 1986, S. 149-170

Hoensch, Jörg K., Geschichte der Tschechoslowakischen Republik 1918-1978, Stuttgart u. a. 21978

Holl, Karl/Trautmann, Günter/Vorländer, Hans (Hg.), Sozialer Liberalismus, Göttingen 1986

Hoock, Jochen, Le problème de la réforme de l'Etat en France, 1880-1935, Thèse FNSP Paris 1970

Hornung, Klaus, Der Jungdeutsche Orden (Beiträge zur Geschichte des Parlamentarismus und der politischen Parteien 14), Düsseldorf 1958

Huard, Raymond, Le suffrage universel en France (1848-1946), Paris 1991

Ders., La naissance du parti politique en France, Paris 1996

Hudemann, Rainer, Fraktionsbildung im französischen Parlament. Zur Entwicklung des Parteiensystems in der frühen Dritten Republik (1871-1875), München 1979

Ders., Politische Reform und gesellschaftlicher status quo. Thesen zum französischen Liberalismus im 19. Jahrhundert, in: Dieter Langewiesche (Hg.), Liberalismus im 19. Jahrhundert. Deutschland im europäischen Vergleich (Kritische Studien zur Geschichtswissenschaft 79), S. 332-352 
Ders., Reparationsgut oder Partner? Zum Wandel in der Forschung über Frankreichs Deutschlandpolitik nach 1945, in: Aus Politik und Zeitgeschichte. Beilage zur Wochenzeitung Das Parlament B 6/97 v. 31. 1. 1997, S. 31-40

Ders./Kaelble, Hartmut/Schwabe, Klaus (Hg.), Europa im Blick der Historiker. Europäische Integration im 20. Jahrhundert: Bewußtsein und Institutionen (Historische Zeitschrift Beihefte N.F. 21), München 1995

Hüser, Dietmar, Frankreichs „doppelte Deutschlandpolitik“. Dynamik aus der Defensive Planen, Entscheiden, Umsetzen in gesellschaftlichen und wirtschaftlichen, innen- und außenpolitischen Krisenzeiten 1944-1950, Berlin 1996

Hughes, Judith M., To the Maginot Line. The Politics of French Military Preparation in the 1920's, Cambridge/Mass. 1971

Irvine, William D., French Conservatism in Crisis. The Republican Federation of France in the 1930s, Baton Rouge/London 1979

Isoart, Paul/Bidegaray Christian (Hg.), Des Républiques françaises, Paris 1988

Jackson, Julian, The politics of depression in France 1932-1936, Cambridge 1985

Ders., The Popular Front in France defending democracy, 1934-38, Cambridge 1988

Jacobson, Jon, Locarno Diplomacy. Germany and the West, 1925-1929, Princeton 1972

Jacques, Léon, Les partis politiques sous la IIIle République, Paris 1913

Jäckel, Eberhard, Frankreich in Hitlers Europa. Die deutsche Frankreichpolitik im Zweiten Weltkrieg (Quellen und Darstellungen zur Zeitgeschichte 14), Stuttgart 1966

Jardin, André, Histoire du libéralisme politique de la crise de l'absolutisme à la constitution de 1875, Paris 1985

Jeanneney, Jean-Noël, François de Wendel en République. L'argent et le pouvoir 1914-1940, Paris 1976

Ders., Leçon d'histoire pour une gauche au pouvoir. La faillite du Cartel 1924-1926, Paris 1977

Ders, La politique économique de Léon Blum, in: Renouvin/Rémond ( $\mathrm{Hg}$.), Léon Blum, Chef de gouvernement 1936-1937, S. 207-232

Jolly, Jean (Hg.), Dictionnaire des parlementaires français. Notices biographiques sur les ministres, députés et sénateurs français de 1889 à 1940, 8 Bände, Paris 1960-1977

Judt, Tony, La reconstruction du parti socialiste, 1921-1926, Paris 1976

Kaelble, Hartmut, Nachbarn am Rhein. Entfremdung und Annäherung der französischen und deutschen Gesellschaft seit 1880, München 1991

Kammerer, Albert, La vérité sur l'armistice. Ephéméride de ce qui s'est réellement passé au moment du désastre, Paris 1944

Kemp, Tom, The French Economy, 1913-39. The History of a Decline, London 1972

Kent, Bruce, The Spoils of War. The Politics, Economics, and Diplomacy of Reparations, 1918-1932, Oxford 21991

Kersaudy, François, 1940. La guerre du fer, Paris 1987

Kessel, Martina, Westeuropa und die deutsche Teilung. Englische und französische Deutschlandpolitik auf den Außenministerkonferenzen von 1945 bis 1947, München 1989

Kettle, Michael, Russia and the Allies 1917-1920. Band II: The Road to Intervention. MarchNovember 1918, London/New York 1988

Kiersch, Gerhard, Parlament und Parlamentarier in der Außenpolitik der IV. Republik, Diss. Berlin 1971

Kindleberger, Charles P., The World in Depression, 1929-1939, Berkeley 1973

Knipping, Franz, Deutschland, Frankreich und das Ende der Locarno-Ära 1928-1931. Studien zur internationalen Politik in der Anfangsphase der Weltwirtschaftskrise, München 1987

Köhler, Henning, Novemberrevolution und Frankreich. Die französische Deutschlandpolitik 1918-1919, Düsseldorf 1980

Kolár, Frantisek, Edouard Beneš et le Conseil national tchécoslovaque, in: Guerres mondiales et conflits contemporains 169 (1993), S. 9-15

Kooker, Judith L., French Financial Diplomacy. The Interwar Years, in: Benjamin M. Row- 
land (Hg.), Balance of Power or Hegemony. The Interwar Monetary System, New York 1976, S. 83-145

Korff, Adalbert, Le revirement de la politique française à l'égard de l'Allemagne entre 1945 et 1950, Ambilly-Annemasse 1965

Korpi, Walter, Un Etat-Providence contesté et fragmenté. Le développement de la citoyenneté sociale en France. Comparaisons avec la Belgique, l'Allemagne, l'Italie et la Suède, in: Revue française de science politique 45 (1995), S. 632-667

Kovác, Dusan, Deux hommes dans la Grande Guerre: Maurice Janin et Milan Rastislav Stefánik, in: Guerres mondiales et conflits contemporains 169 (1993), S. 51-58

Krüger, Peter, Deutschland und die Reparationen 1918/19. Die Genesis des Reparationsproblems in Deutschland zwischen Waffenstillstand und Versailler Friedensschluß (Schriftenreihe der Vierteljahrshefte für Zeitgeschichte 25), Stuttgart 1973

Ders., Das Reparationsproblem der Weimarer Republik in fragwürdiger Sicht. Kritische Überlegungen zur neuesten Forschung, in: VfZ 29 (1981), S. 21-47

Ders., Die Auswirkungen der Inflation auf die deutsche Außenpolitik, in: Gerald D. Feldman (Hg.), Die Nachwirkungen der Inflation auf die deutsche Geschichte 1924-1933 (Schriften des Historischen Kollegs, Kolloquien 6), München 1985, S. 297-314

Ders., Die Außenpolitik der Republik von Weimar, Darmstadt ${ }^{2} 1993$

Krumeich, Gerd, Aufrüstung und Innenpolitik in Frankreich vor dem Ersten Weltkrieg. Die Einführung der dreijährigen Dienstpflicht 1913-1914 (Veröffentlichungen des Instituts für Europäische Geschichte Mainz, Band 96), Wiesbaden 1980

Ders., Der politische Liberalismus im parlamentarischen System Frankreichs vor dem Ersten Weltkrieg, in: Dieter Langewiesche ( $\mathrm{Hg}$.), Liberalismus im 19. Jahrhundert. Deutschland im europäischen Vergleich (Kritische Studien zur Geschichtswissenschaft 79), Göttingen 1988 , S. 353-366

Küsters, Hanns Jürgen, Die Gründung der Europäischen Wirtschaftsgemeinschaft, BadenBaden 1982

Kuisel, Richard F., Capitalism and the state in modern France. Renovation and economic management in the twentieth century, Cambridge 1983

Kupferman, Fred/Machefer, Philippe, Presse et politique dans les années trente: le cas du ,Petit Journal', in: Revue d'histoire moderne et contemporaine 22 (1975), S. 7-51

Lacaze, Yvon, L'opinion publique française et la crise de Munich, Frankfurt u. a. 1991

Ders., L'opinion publique française et la crise de Munich, in: Francia 18/3 (1991), S. $73-83$

Ders., La France et Munich. Etude d'un processus décisionnel en matière de relations internationales (Collections contacts, Série II: Gallo-Germanica 8), Bern 1992

Lachapelle, Georges, Elections législatives des 26 avril et 10 mai 1914, Paris 1914

Ders., Elections législatives du 16 novembre 1919. Résultats officiels, Paris 1920

Ders., Elections Législatives. 22-29 avril 1928. Résultats Officiels, Paris 1928

Ders., L'Alliance démocratique. Ses origines, ses hommes, son rôle, Paris 1935

Lacour-Gayet, Robert, Le problème de la dette française envers les Etats-Unis après la Première Guerre mondiale (1917-1932), in: Revue d'histoire diplomatique 75 (1961), S. 10-24

Lacouture, Jean, Léon Blum, Paris 1977

Ders., Charles de Gaulle. Band I: Le rebelle. 1890-1944, Paris 1984; Band II: Le politique. 1944-1959, Paris 1985; Band III: Le souverain. 1959-1970, Paris 1986

Langewiesche, Dieter, Die Anfänge der deutschen Parteien. Partei, Fraktion und Verein in der Revolution von 1848/49, in: Geschichte und Gesellschaft 4 (1978), S. 324-361

Ders. (Hg.), Liberalismus im 19. Jahrhundert. Deutschland im europäischen Vergleich (Kritische Studien zur Geschichtswissenschaft 79), Göttingen 1988

Ders., Deutscher Liberalismus im europäischen Vergleich: Konzeption und Ergebnisse, in: Ders. (Hg.), Liberalismus im 19. Jahrhundert, S. 11-19

Ders., Liberalismus in Deutschland, Frankfurt a. M. 1988

Ders., Republik und Republikaner. Von der historischen Entwertung eines politischen Begriffs (Stuttgarter Vorträge zur Zeitgeschichte 1), Essen 1993 
La Pradelle, Paul de, La réforme de l'Etat français. Essai de bibliographie synthétique, in: Annales du droit et des sciences sociales 2 (1934), S. 393-448

Larkin, Maurice, Church and State after the Dreyfus Affair. The Separation Issue in France, London 1974

Latour, Jean, Paul Reynaud, Paris 1946

Le Béguec, Gilles, L'entrée au Palais-Bourbon: Les filières privilégiées d'accès à la fonction parlementaire (1919-1939). Unveröff. Thèse d'Etat, Paris X - Nanterre 1989

Ders., L’aristocratie du barreau, vivier pour la République. Les secrétaires de la Conférence du stage, in: Vingtième Siècle 30 (1991), S. 22-31

Ders., Le parti, in: Jean-François Sirinelli (Hg.), Histoire des droites en France. Band II, Paris 1992, S. 13-59

Ders., De la république des avocats à la république des énarques, in: Louis Dupeux/Rainer Hudemann/Franz Knipping (Hg.), Eliten in Deutschland und Frankreich im 19. und 20. Jahrhundert. Strukturen und Beziehungen. Elites en France et en Allemagne aux XIXème et XXème siècles. Structures et relations, Band II, München 1996, S. 79-89

Lefranc, Georges, Le syndicalisme en France, Paris ${ }^{9} 1975$

Le Van-Lemesle, Lucette, La perception de l'innovation chez les économistes français (18961939), in: Revue Historique 271 (1984), S. 369-376

Lévêque, Pierre, Histoire des forces politiques en France. Band II: 1880-1940, Paris 1994

Leyret, Henry, Waldeck-Rousseau et la Troisième République (1869-1889), Paris 1908

Lichtenberger, Henri, L'Allemagne d'aujourd'hui dans ses relations avec la France, Paris 1922

Lipgens, Walter, Die Anfänge der europäischen Einigungspolitik 1945-1950. I. Teil: 19451947, Stuttgart 1977

Logue, William, From Philosophy to Sociology. The Evolution of French Liberalism, 18701914, DeKalb/Illinois 1983

Loth, Wilfried, Sozialismus und Internationalismus. Die französischen Sozialisten und die Nachkriegsordnung Europas 1940-1950, Stuttgart 1977

Ders., Die Franzosen und die deutsche Frage 1945-1949, in: Claus Scharf/Hans-Jürgen Schröder (Hg.), Die Deutschlandpolitik Frankreichs und die französische Zone 19451949, Wiesbaden 1983, S. 27-48

Ders., Geschichte Frankreichs im 20. Jahrhundert, Stuttgart u.a. 1987

Ders., Die deutsche Frage in französischer Perspektive, in: Ders., Ost-West-Konflikt und deutsche Frage, München 1989, S. 46-64

Ders., Der Weg nach Europa. Geschichte der europäischen Integration 1939-1957, Göttingen 1990

Lottman, Herbert R., L'Epuration 1943-1953, Paris 1986

Loubet del Bayle, Jean-Louis, Les non-conformistes des années trente, Paris 1969

Lovighi, Christian, Henri de Kerillis (1889-1950), Thèse IEP Paris 1992

Machefer, Philippe, Ligues et fascismes en France (1919-1939), Paris 1974

Magraw, Roger, Workers and the bourgeois republic (A history of the French working class 2), Oxford 1992

Discours de Mlle. Valérie Maintrieu (Audience solennelle de rentrée de la Conférence du stage, 29 juin 1984), in: La Gazette du Palais, 4 au 6 novembre 1984, S. 6-12

Margairaz, Michel, L'Etat, les finances et l'économie. Histoire d'une conversion 1932-1952. 2 Bände, Paris 1991

Marin, Louis, Contribution à l'étude des prodromes de l'armistice, in: Revue d'Histoire de la Deuxième Guerre Mondiale 1 (1951), S. 1-26

Ders., Gouvernement et commandement. Conflits, différends, immixtions qui ont pesé sur l'armistice de juin 1940, in: Revue d'histoire de la deuxième guerre mondiale 2/8 (1952), S. 1-28 sowie 3/9 (1953), S. 1-14

Marseille, Jacques, Les origines ,inopportunes' de la crise de 1929 en France, in: Revue économique 31 (1980), S. 648-684

Ders., Empire colonial et capitalisme français. Histoire d'un divorce, Paris 1984 
Ders., Colonisation, décolonisation et capitalisme (1880-1960). Un divorce à la française, in: Vingtième Siècle 4 (1984), S. 39-48

Ders., Crise de 1929, in: Jean-François Sirinelli (Hg.), Dictionnaire historique de la vie politique française au XXe siècle, Paris 1995, S. 238-241

Marshall, D. Bruce, The French Colonial Myth and Constitution-Making in the Fourth Republic, New Haven/London 1973

Martens, Stefan, Einleitung, in: Ders. (Hg.), Documents Diplomatiques Français sur l'Allemagne 1920. Französische Diplomatenberichte aus Deutschland 1920, Band I (Pariser Historische Studien 33/1), Bonn/Berlin 1992, S. 29-149

Ders., Alexandre Millerand. Der Mann zwischen Clemenceau und Poincaré, in: Historische Mitteilungen der Ranke-Gesellschaft 5 (1992), S. 96-113

Ders. (Hg.), Vom „Erbfeind“ zum „Erneuerer“. Aspekte und Motive der französischen Deutschlandpolitik nach dem Zweiten Weltkrieg, Sigmaringen 1993

Martin, Bernd, Friedensinitiativen und Machtpolitik während des Zweiten Weltkrieges 1939-1942, Düsseldorf 1974

Martin, Olivier, Les institutions et les lois de la Troisième République, Paris 1936

Martinaud-Déplat, Léon, Un parlementaire, in: La Revue des Deux Mondes 21 (1966), S. 3-8

Martinez, Gilles, Le „Centre national des indépendants“ de 1948 à 1962, Thèse Paris 1992

Maurois, André, The battle of France, London 1941

Mayer, Arno J., Politics and Diplomacy of Peacemaking. Containment and Counterrevolution at Versailles, 1918-1919, London 1968

Mayeur, Jean-Marie, La vie politique sous la Troisième République, Paris 1984

Ders., Les débuts de la Troisième République, 1871-1898, Paris 1973

McDougall, Walter A., France's Rhineland Diplomacy, 1914-1924. The Last Bid for a Balance of Power in Europe, Princeton 1978

McManners, John, Church and State in France, 1870-1914, London 1972

Merglen, Albert, Quelques réflexions historiques sur l'armistice franco-germano-italien de juin 1940, in: Guerres mondiales et conflits contemporains 45 (1995), S. 79-93

Micaud, Charles, The French Right and Nazi Germany 1933-1939. A Study of Public Opinion, New York 1964

Michael, Robert, The Radicals and Nazi Germany. The Revolution in French Attitudes toward Foreign Policy, 1933-1939, Washington 1982

Michel, Henri, La drôle de guerre, Paris 1971

Ders., Le procès de Riom, Paris 1979

Miller Unterberger, Betty, Woodrow Wilson and the Russian Revolution, in: Arthur B. Link (Hg.), Woodrow Wilson and a Revolutionary World, 1913-1921, Chapel Hill 1982, S. 49104

Dies., The United States, Revolutionary Russia, and the Rise of Czechoslovakia, Chapel Hill/London 1989

Millin de Grandmaison, Georges, Les conseils économiques français. Leur genèse et leur évolution, Thèse Paris 1962

Milza, Pierre, Fascisme français. Passé et présent, Paris 1987

Miquel, Pierre, Poincaré, Paris 1961

Ders., Les quatre vingts. Ils ont dit NON à Pétain le 10 juillet 1940, Paris 1995

Möller, Horst, Parlamentarismus in Preußen 1919-1932, Düsseldorf 1985

Ders., Parlamentarismus-Diskussion in der Weimarer Republik: Die Frage des , besonderen“ Weges zum parlamentarischen Regierungssystem, in: Manfred Funke u.a. (Hg.), Demokratie und Diktatur. Geist und Gestalt politischer Herrschaft in Deutschland und Europa. Festschrift für Karl Dietrich Bracher, Düsseldorf 1987, S. 140-157

Ders., Bürgertum und bürgerlich-liberale Bewegung nach 1918, in: Lothar Gall (Hg.), Bürgertum und bürgerlich-liberale Bewegung in Mitteleuropa seit dem 18. Jahrhundert (HZSonderheft 17), S. 293-342 
Ders., Europa zwischen den Kriegen (Oldenbourg Grundriß der Geschichte 21), München 1998

Molènes, Charles Melchior de, L'Europe de Strasbourg. Une première expérience de parlementarisme européen, Paris 1971

Moneta, Jakob, Die Kolonialpolitik der französischen KP, Hannover 1968

Monnet, François, Refaire la République. André Tardieu, une dérive réactionnaire (18761945), Paris 1993

Montagnon, Pierre, La France coloniale. La gloire de l'Empire, Paris 1988

Moreau, Félix, Régime parlementaire et principe représentatif, in: Revue politique et parlementaire 27 (1901), S. $331 \mathrm{ff}$. und 28 (1901), S. $61 \mathrm{ff}$.

Ders., Précis élémentaire de droit constitutionnel, Paris ${ }^{7} 1911$

Mouraz, Bernard, L'Alliance Démocratique et la Guerre 1914-1919, Mémoire de Maitrise, Université Paris I 1971

Mouré, Kenneth, Managing the franc Poincaré. Economic understanding and political constraint in French monetary policy, 1928-1936, Cambridge 1991

Mühle, Robert W., Frankreich und Hitler. Die französische Deutschland- und Außenpolitik 1933-1935, Paderborn u. a. 1995

Ders., Louis Barthou und Deutschland (1862-1934), in: Francia 21/3 (1994), S. 71-98

Müller, Klaus-Jürgen, Das Ende der Entente Cordiale. Eine Studie zur Entwicklung der englisch-französischen Beziehungen während des Westfeldzuges 1940 (Beihefte der Wehrwissenschaftlichen Rundschau 3), Frankfurt a. M. 1956

Ders., Zum deutsch-französischen Waffenstillstand 1940, in: Wehrwissenschaftliche Rundschau 11 (1961), S. 356-359

Ders., Protest - Modernisierung - Integration. Bemerkungen zum Problem faschistischer Phänomene in Frankreich 1924-1934, in: Francia 8 (1980), S. 465-524

Munch-Petersen, Thomas, The Strategy of Phoney War. Britain, Sweden and the Iron Ore Question 1939-1940, Stockholm 1981

Mysyrowicz, Ladislas, Autopsie d'une défaite. Origines de l'effondrement militaire français de 1940, Paris 1973

Neave-Hill, W.B.R., L'évolution de la stratégie franco-anglaise (1939-1940), in: Français et Britanniques dans la Drôle de guerre, S. 333-358

Nechvatal, Martin, La naissance d'une armée tchécoslovaque en France, in: Guerres mondiales et conflits contemporains 169 (1993), S. 37-41

Nehring, Walther K., Die Geschichte der deutschen Panzerwaffe 1916 bis 1945, Berlin 1969

Nicolet, Claude, Le radicalisme, Paris 1967

Ders., L'idée républicaine en France (1789-1924). Essai d'histoire critique, Paris 1982

Ders., La République en France. Etat des lieux, Paris 1992

Nipperdey, Thomas, Einführung, in: Langewiesche (Hg.), Liberalismus im 19. Jahrhundert, S. $279-281$

Ders., Die Organisation der bürgerlichen Parteien in Deutschland vor 1918, in: HZ 185 (1958), S. 550-602

Ders., Die Organisation der deutschen Parteien vor 1918, Düsseldorf 1961

Nordmann, Jean-Thomas, Histoire des Radicaux, 1820-1973, Paris 1974

Ders., La France radicale, Paris 1977

Ohler, Norbert, Deutschland und die deutsche Frage in der „Revue des deux Mondes“ 19051940. Ein Beitrag zur Erhellung des französischen Deutschlandbildes, Frankfurt 1973

Orde, Anne, British Policy and European Reconstruction after the First World War, Cambridge 1990

Ottmer, Hans-Martin, „Weserübung“. Der deutsche Angriff auf Dänemark und Norwegen im April 1940 (Operationen des Zweiten Weltkrieges 1), München 1994

Parker, R.A.C., Grande-Bretagne, France et Scandinavie en 1939-1940: la politique, les hommes et la stratégie, in: Français et Britanniques dans la Drôle de guerre, S. 561-582

Pasemann, Martin Ernst, Paul Reynaud, in: Zeitschrift für Politik 4/5 (1940), S. 203-207

Patat, Jean-Pierre/Lutfalla, Michel, Histoire monétaire de la France au XXe siècle, Paris 1986 
Paulhac, François, Les accords de Munich et les origines de la guerre de 1939, Paris 1988

Paxton, Robert O., La France de Vichy 1940-1944, Paris ${ }^{2} 1997$

Payen, Fernand/Duveau, Gaston, Les règles de la profession d'avocat et les usages du barreau de Paris, Paris ${ }^{2} 1926$

Pereboom, Maarten L., Democracies at the Turning Point. Britain, France and the End of the Postwar Order, 1928-1933 (Studies in Modern European History 13), New York u.a. 1995

Pertinax [Géraud, André], Les Fossoyeurs. La bataille de France, l'armistice, la contre-révolution. Band I: Les derniers chefs de la IIIe République. Gamelin, Paul Reynaud, Daladier, Paris 1946; Band II: Pétain, Paris 1946

Petit, Lucien, Histoire des finances extérieures de la France. Le règlement des dettes interalliées, 1919-1929, Paris 1932

Pinol, Jean-Luc, 1919-1958. Le temps des droites?, in: Jean-François Sirinelli (Hg.), Histoire des droites en France, Paris 1992, S. 291-389

Pirou, Gaetan, La monnaie française depuis la guerre, 1914-1936. Inflation, stabilisation, dévaluation, Paris 1936

Plaisant, Marcel, Un portrait inédit de Paul Reynaud, in: Revue politique et parlementaire 68 (1966), S. 38-48

Poidevin, Raymond, Die französische Deutschlandpolitik 1943-1949, in: Claus Scharf/ Hans-Jürgen Schröder ( $\mathrm{Hg}$.), Die Deutschlandpolitik Frankreichs und die französische Zone 1945-1949, Wiesbaden 1983, S. 15-25

Ders., Frankreich und die deutsche Frage 1943-1949, in: Josef Becker/Andreas Hillgruber (Hg.), Die deutsche Frage im 19. und 20. Jahrhundert, München 1983, S. 405-420

Ders., Frankreich und das Problem der EVG: Nationale und internationale Einflüsse (Sommer 1951 bis Sommer 1953), in: Hans-Erich Volkmann/Walter Schwengler (Hg.), Die Europäische Verteidigungsgemeinschaft. Stand und Probleme der Forschung, Boppard am Rhein 1985, S. 101-124

Ders., Robert Schuman. Homme d'Etat, 1886-1963, Paris 1986

Ders., Vers une relance des relations économiques franco-allemandes en 1938-1939, in: Hildebrand/Werner (Hg.), Deutschland und Frankreich 1936-1939, S. 351-363; wiederabgedruckt in: Poidevin, Raymond, Péripéties franco-allemandes. Du milieu du XIXe siècle aux années 1950. Recueil d'articles, Bern u.a. 1995, S. 259-273

Ders. (Hg.), Histoire des débuts de la construction européenne (Mars 1948-mai 1950). Origins of the European Integration (March 1948-may 1950). Actes du colloque de Strasbourg 28-30 novembre 1984, Bruxelles/Milano/Paris/Baden-Baden 1986

Posen, Barry R., The Sources of Military Doctrine. France, Britain, and Germany between the World Wars, Ithaca/London 1984

Prost, Antoine, Les anciens combattants et la société française (1914-1939), 3 Bände, Paris 1977

Ders., Le climat social, in: Rémond/Bourdin (Hg.), Edouard Daladier, S. 99-111

Ders., Die Demobilmachung, der Staat und die Kriegsteilnehmer in Frankreich, in: Geschichte und Gesellschaft 9 (1983), S. 178-194

Réau, Elisabeth du, L'aménagement de la loi instituant la semaine de quarante heures, in: Rémond/Bourdin (Hg.), Edouard Daladier, S. 129-149

Dies., Edouard Daladier. 1884-1970, Paris 1993

Rebérioux, Madeleine, La République radicale? 1898-1914 (Nouvelle histoire de la France contemporaine 11), Paris 1975

De la récurrence des crises économiques. X-Crise. Centre Polytechnicien d'Etudes Economiques. Son Cinquantenaire 1931-1981, Paris 1982

Les réformes politiques de la France. Conférences faites à l'Ecole des Hautes Etudes sociales, Paris 1924

Rémond, René, Les Etats-Unis devant l'opinion française 1815-1852, Paris 1962

Ders., Introduction, in: Ders./Bourdin (Hg.), Edouard Daladier, S. 9-21

Ders., Les droites en France, Paris 1982 
Ders., La fin de la IIIe République, in: Paul Isoart/Christian Bidegaray (Hg.), Des Républiques françaises, Paris 1988, S. 602-609

Ders., Frankreich im 20. Jahrhundert. Erster Teil 1918-1958, Stuttgart 1994

Ders./Bourdin, Janine (Hg.), Edouard Daladier, Chef de gouvernement, avril 1938 - septembre 1939, Paris 1977

Diess. (Hg.), La France et les Français en 1938/1939, Paris 1978

Diess., Les forces adverses, in: Pierre Renouvin/René Rémond (Hg.), Léon Blum, Chef de gouvernement 1936-1937, S. 137-159

Renouvin, Pierre/Rémond, René (Hg.), Léon Blum, Chef de gouvernement 1936-1937 (Cahiers de la Fondation Nationale des Sciences Politiques 155), Paris 1967

Rials, Stéphane, Textes constitutionnels français, Paris 101994

Rioux, Jean-Pierre, La France de la Quatrième République. Band I: L'ardeur et la nécessité 1944-1952, Paris 1980; Band II: L'expansion et l'impuissance 1952-1958, Paris 1983

Ders., Französische öffentliche Meinung und EVG: Parteienstreit oder Schlacht der Erinnerungen?, in: Hans-Erich Volkmann/Walter Schwengler (Hg.), Die Europäische Verteidigungsgemeinschaft. Stand und Probleme der Forschung, Boppard am Rhein 1985, S. 159176

Ders., L'association en politique, in: René Rémond ( $\mathrm{Hg}$.), Pour une histoire politique, Paris 1988 , S. 87-120

Roberts, Stephen H., The History of French Colonial Policy 1870-1925, London 1963

Rohe, Karl, Das Reichsbanner Schwarz-Rot-Gold. Ein Beitrag zur Geschichte und Struktur der politischen Kampfverbände zur Zeit der Weimarer Republik (Beiträge zur Geschichte des Parlamentarismus und der politischen Parteien 34), Düsseldorf 1966

Ders., Parteien und Parteiensystem, in: Hans Kastendiek/Karl Rohe/Angelika Volle (Hg.), Großbritannien. Geschichte, Politik, Wirtschaft, Gesellschaft, Frankfurt/New York 1995, S. 213-229

Rossi-Landi, Guy, La Drôle de guerre. La vie politique en France, 2 septembre 1939 - 10 mai 1940, Paris 1971

Rossiter, Adrian, Tardieu, André, in: David S. Bell/Douglas Johnson/Peter Morris (Hg.), Biographical Dictionary of French Political Leaders since 1870, New York u.a. 1990, S. $408-410$

Rothermund, Dietmar (Hg.), Die Peripherie in der Weltwirtschaftskrise: Afrika, Asien und Lateinamerika 1929-1939, Paderborn 1983

Roussellier, Nicolas, André Tardieu et la crise du constitutionnalisme libéral (1933-1934), in: Vingtième Siècle 21 (1989), S. 57-70

Ders., L'Europe des libéraux, Paris 1991

Ders., Tardieu André, in: Jean-François Sirinelli (Hg.), Dictionnaire historique de la vie politique française au XXe siècle, Paris 1995, S. 1001-1003

Ders., Libéralisme, in: Jean-François Sirinelli (Hg.), Dictionnaire historique de la vie politique française au XXe siècle, Paris 1995, S. 585-589

Ders., Le parlement de l'éloquence. La souveraineté de la délibération au lendemain de la Grande Guerre, Paris 1997

Rousso, Henry, L'épuration en France. Une histoire inachevée, in: Vingtième Siècle 33 (1992), S. 78-105

Rupieper, Hermann, The Cuno government and reparations 1922-1923. Politics and economics, Den Haag u. a. 1979

Sablier, Edouard, La création du „Monde“, Paris 1984

Sagnes, Jean, Le refus républicain: Les quatre-vingts parlementaires qui dirent „non“ à Vichy le 10 juillet 1940, in: Revue d'histoire moderne et contemporaine 38 (1991), S. 555-589

Salewski, Michael, Knotenpunkt der Weltgeschichte? Die Raison des deutsch-französischen Waffenstillstands vom 22. Juni 1940, in: Claude Carlier u.a. (Hg.), La France et l'Allemagne en guerre, Paris 1990, S. 115-129

Saly, Pierre, La politique des Grands travaux en France, 1929-1939, New York 1977 
Ders., La politique française des grands travaux 1929-1939 fut-elle keynésienne?, in: Revue économique 31 (1980), S. 706-742

Sanson, Rosemonde, L'Alliance démocratique, in: René Rémond/Janine Bourdin ( $\mathrm{Hg}$.), La France et les Français en 1938/1939, Paris 1978, S. 327-339

Dies., L'Alliance démocratique et la perception de la puissance en France en 1938-1939, in: Revue d'Histoire Moderne et Contemporaine 31 (1984), S. 658-666

Dies., Louis Barthou, leader de l'Alliance démocratique, in: Michel Papy (Hg.), Barthou, un homme, une époque. Actes du Colloque de Pau, 9 et 10 novembre 1984, Pau 1987, S. 303315

Dies., Adolphe Carnot et l'Alliance républicaine démocratique, in: Gilles Le Béguec u.a. (Hg.), Une lignée républicaine. Les Carnot sous la Troisième République. Actes du Colloque de Limoges, novembre 1987, Paris 1989, S. 85-103

Dies., Centre et Gauche (1901-1914): L’Alliance Républicaine Démocratique et le Parti radical-socaliste, in: Revue d'Histoire Moderne et Contemporaine 39 (1992), S. 493-512

Dies., ARD ou AD (Alliance démocratique), in: Jean-François Sirinelli (Hg.), Dictionnaire historique de la vie politique française au XXe siècle, Paris 1995, S. 48-53

Sarraut, Albert, La mise en valeur des colonies françaises, Paris 1923

Ders., Grandeur et servitude coloniales, Paris 1931

Sauvy, Alfred, Histoire économique de la France entre les deux guerres. 4 Bände, Paris 19651975

Ders., De Paul Reynaud à Charles de Gaulle. Un économiste face aux hommes politiques 1934-1967. Scènes, tableaux et souvenirs, Paris 1972

Ders., L'évolution économique, in: Rémond/Bourdin (Hg.), Edouard Daladier, S. 87-98

Scherer, Werner, Verhältniswahl und Reform der politischen Ordnung. Die Auseinandersetzung um den Proporz in Frankreich bis zur Wahlreform von 1919, Diss. Heidelberg 1982

Schirmann, Sylvain, Les relations économiques et financières franco-allemandes, 24 décembre 1932 - 1er septembre 1939, Paris 1995

Schmidt, Bernhard u. a. (Hg.), Frankreich-Lexikon. Band 2, Berlin 1983

Schnur, Roman, Das Ende einer Republik. 10. Juli 1940 in Vichy, in: Friedrich J. Kroneck/ Thomas Oppermann (Hg.), Im Dienste Deutschlands und des Rechtes. Festschrift für Wilhelm G. Grewe zum 70. Geburtstag am 16. Oktober 1981, Baden-Baden 1981, S. 337364

Schöttler, Peter, Die Entstehung der „Bourses du Travail“. Sozialpolitik und französischer Syndikalismus am Ende des 19. Jahrhunderts, Frankfurt/New York 1982

Schor, Ralph, Une étude d'opinion: la droite française face à la crise mondiale de 1929, in: L'Information historique 1 (1974), S. 23-28 und 2 (1974), S. 64-70

Ders., Les travailleurs allemands et la reconstruction de la France au lendemain de la Grande Guerre (1919-1923), in: Revue Historique 272 (1984), S. 113-130

Schrecker, Ellen, The Hired Money. The French Debt to the United States, 1917-1929, New York 1978

Schuker, Stephen A., The End of French Predominance in Europe. The Financial Crisis of 1924 and the Adoption of the Dawes Plan, Chapel Hill 1976

Schumacher, Alois, Annäherung durch Verstehen. Pierre Viénot und die deutsch-französischen Beziehungen in den zwanziger Jahren, in: Médiations ou le métier de germaniste. Mélanges offerts à Pierre Bertaux, Asnières 1977, S. 271-277

Schunck, Peter, Charles de Gaulle. Ein Leben für Frankreichs Größe, Berlin 1998

Schwabe, Klaus, Der Marshall-Plan und Europa, in: Rainer Hudemann/Hartmut Kaelble/ Klaus Schwabe (Hg.), Europa im Blick der Historiker. Europäische Integration im 20. Jahrhundert: Bewußtsein und Institutionen (Historische Zeitschrift. Beihefte N.F. 21), München 1995, S. 167-189

Servent, Pierre, Le mythe Pétain. Verdun ou les tranchées de la mémoire, Paris 1992

Setzer, Hans, Wahlsystem und Parteienentwicklung in England. Wege zur Demokratisierung der Institutionen 1832 bis 1948, Frankfurt a. M. 1973 
Shirer, William D., Der Zusammenbruch Frankreichs. Aufstieg und Fall der Dritten Republik, München/Zürich 1970

Sick, Klaus-Peter, Vom Neoliberalismus zum Faschismus? Die Entwicklung der politischen Ideen von Alfred Fabre-Luce und Bertrand de Jouvenel 1918-1945, in: Lendemains 66 (1992), S. 59-75

Siebert, Ferdinand, Aristide Briand, 1862-1932. Ein Staatsmann zwischen Frankreich und Europa, Erlenbach u. a. 1973

Siegfried, André, Tableau politique de la France de l'Ouest sous la IIIe République, Paris 1913

Ders., Tableau des partis en France, Paris 1930

Siegfried, André, u. a., L'économie dirigée. Conférences organisées par la Société des Anciens Elèves de l'Ecole libre des Sciences Politiques, Paris 1934

Simard, Marc, Doumergue et la réforme de l'Etat en 1934: La dernière chance de la IIIe République?, in: French Historical Studies 16/3 (1990), S. 576-596

Simonnot, Philippe, Le secret de l'armistice 1940, Paris 1990

Sirinelli, Jean-François (Hg.), Histoire des droites en France. 3 Bände, Paris 1992

Ders. (Hg.), Dictionnaire historique de la vie politique française au XXe siècle, Paris 1995

Sorlin, Pierre, Waldeck-Rousseau, Paris 1966

Soucy, Robert, French Fascism. The First Wave, 1924-1933, New Haven/London 1986

Ders., French Fascism. The Second Wave, 1933-1939, New Haven/London 1995

Soulié, Michel, La vie politique d'Edouard Herriot, Paris 1962

Soutou, Georges, Die deutschen Reparationen und das Seydoux-Projekt 1920/21, in: VfZ 23 (1975), S. 237-270

Stamm, Christoph, Lloyd George zwischen Innen- und Außenpolitik. Die britische Deutschlandpolitik 1921/22, Köln 1977

Stone, Judith F., The Search for Social Peace. Reform Legislation in France, 1890-1914, Albany 1985

Taÿ, Hugues, Le régime présidentiel et la France. Etude d'histoire des idées juridiques et politiques, Paris 1967

Tellier, Thibault, Paul Reynaud 1945-1966. Contribution à une histoire des droites après la Seconde guerre mondiale. Mémoire de DEA, Université de Lille 1992

Ders., La contribution politique de Paul Reynaud aux grands débats nationaux de 1945 à 1966, in: Francia 23/3 (1996), S. 93-111

Thobie, Jacques/Meynier, Gilbert/Coquery-Vidrovitch, Catherine/Ageron, Charles-Robert, Histoire de la France coloniale. Band II: 1914-1990, Paris 1990

Thunig-Nittner, Gerburg, Die tschechoslowakische Legion in Rußland. Ihre Geschichte und Bedeutung bei der Entstehung der 1. Tschechoslowakischen Republik (Marburger Ostforschungen 30), Wiesbaden 1970

Tiemann, Dieter, Der Jungdeutsche Orden und Frankreich, in: Francia 12 (1984), S. 425456

Touchard, Jean, La gauche en France depuis 1900, Paris 21981

Ders., Histoire des idées politiques. Band II: Du XVIIIe siècle à nos jours, Paris ${ }^{6} 1973$

De la Tour-du-Pin, Hadelin, La presse de droite contre la dévaluation: Sa campagne contre Paul Reynaud, Mémoire de Maîtrise, Paris 1972

Tournoux, Paul-Emile, Défense des frontières. Haut commandement-gouvernement, 19191939, Paris 1960

Trachtenberg, Marc, Reparation in World Politics. France and European Economic Diplomacy, 1916-1923, New York 1980

Trausch, Gilbert (Hg.), Die europäische Integration vom Schuman-Plan bis zu den Verträgen von Rom. The European Integration from the Schuman-Plan to the Treaties of Rome. Pläne und Initiativen, Enttäuschungen und Mißerfolge. Projects and Initiatives, Disappointments and Failures. Beiträge des Kolloquiums in Luxemburg 17.-19. Mai 1989. Contributions to the Symposium in Luxemburg May 17-19, 1989, Baden-Baden/Milano/Paris/Bruxelles 1993 
Truchet, André, L'Armistice de 1940 et l'Afrique du Nord, Paris 1955

Vavasseur-Desperriers, Jean, République et liberté. Charles Jonnart, une conscience républicaine (1857-1927), Villeneuve-d'Ascq 1996

Vidal, Christiane, Chronologie et rythmes du dépeuplement dans le département des Alpes de Haute-Provence depuis le début du XIXe siècle, in: Provence Historique 21 (1971), S. 281-291

Viel, Jean, Paul Reynaud, Paris 1940

Vietsch, Eberhard von, Arnold Rechberg und das Problem der politischen West-Orientierung Deutschlands nach dem 1. Weltkrieg, Koblenz 1958

Wandycz, Piotr S., France and Her Eastern Allies 1919-1925. French-Czechoslovak-Polish Relations from the Paris Peace Conference to Locarno, Minneapolis 1962

Ders., The Twilight of French Eastern Alliances, 1926-1936. French-Czechoslovak-Polish Relations from Locarno to the Remilitarization of the Rhineland, Princeton 1988

Weber, Eugen, L'Action française, Stanford 1962

Ders., La France des années 30. Tourments et perplexités, Paris 1995

Wehner, Gerd, Die Westalliierten und das Grundgesetz 1948-1949. Die Londoner Sechsmächtekonferenz, Freiburg im Br. 1994

Weill-Raynal, Etienne, Les réparations allemandes et la France, 3 Bände, Paris 1947

Weinreis, Hermann, Liberale oder autoritäre Republik. Regimekritik und Regimekonsens der französischen Rechten zur Zeit des nationalsozialistischen Aufstiegs in Deutschland (1928-1934) (Göttinger Bausteine zur Geschichtswissenschaft 54), Göttingen/Zürich 1986

Weisenfeld, Ernst, Welches Deutschland soll es sein? Frankreich und die deutsche Einheit seit 1945, München 1986

Weiß, Hermann/Hoser, Paul (Hg.), Die Deutschnationalen und die Zerstörung der Weimarer Republik. Aus dem Tagebuch von Reinhold Quaatz 1928-1933 (Schriftenreihe der Vierteljahrshefte für Zeitgeschichte 59), München 1989

Wheatley, Ronald, La guerre russo-finlandaise, les plans d'intervention alliés et les relations britanniques avec la Russie, in: Français et Britanniques dans la Drôle de guerre, S. 245261

Wieland, Volker, Zur Problematik der französischen Militärpolitik und Militärdoktrin in der Zeit zwischen den Weltkriegen (Militärgeschichtliche Studien 15), Boppard am Rhein 1973

Wileman, Donald G., L'Alliance Républicaine Démocratique. The Dead Centre of French Politics, 1901-1947, Downsview/Ontario 1988

Ders., Pierre-Etienne Flandin and the Alliance Démocratique, 1929-1939, in: French History 4 (1990), S. 139-173

Williams, Philip, La vie politique sous la 4 e République, Paris 1971

Willis, F. Roy, France, Germany, and the New Europe 1945-1963, Stanford/London 1965

Winock, Michel, Le mythe fondateur: l'affaire Dreyfus, in: Berstein/Rudelle ( $\mathrm{Hg}$.), Modèle républicain, S. 131-145

Ders., Nationalisme, antisémitisme et fascisme en France, Paris 1990

Wirsching, Andreas, Vom Weltkrieg zum Bürgerkrieg? Politischer Extremismus in Deutschland und Frankreich 1918-1933/39. Berlin und Paris im Vergleich (Quellen und Darstellungen zur Zeitgeschichte 40), München 1999

Ders., Kleinbürger für den Klassenkampf? Theorie und Praxis kommunistischer Mittelstandspolitik in Frankreich 1924-1936, in: Horst Möller/Gérard Raulet/Andreas Wirsching (Hg.), Gefährdete Mitte? Mittelschichten und politische Kultur zwischen den Weltkriegen: Italien, Frankreich und Deutschland, Sigmaringen 1993, S. 95-116

Ders., Großbritanniens Europapolitik und das deutsch-französische Problem nach den beiden Weltkriegen, in: GWU 4 (1996), S. 209-224

Wolgast, Eike, Reform, Reformation, in: Geschichtliche Grundbegriffe. Historisches Lexikon zur politisch-sozialen Sprache in Deutschland. Hg. von Otto Brunner, Werner Conze und Reinhard Koselleck, Stuttgart 1984

Wolikow, Serge, Le Front populaire en France, Paris 1996 
Wormser, Georges, Le septennat de Poincaré, Paris 1977

Wulf, Peter, Hugo Stinnes. Wirtschaft und Politik 1918-1924, Stuttgart 1979

Wurm, Clemens A., Die französische Sicherheitspolitik in der Phase der Umorientierung 1924-1926, Frankfurt a.M. u.a. 1979

Ders., Frankreich, die Reparationen und die interalliierten Schulden in den 20er Jahren, in: Gerald D. Feldman (Hg.), Die Nachwirkungen der Inflation auf die deutsche Geschichte 1924-1933 (Schriften des Historischen Kollegs, Kolloquien 6), München 1985, S. 315-334

Ders., Westminster als Modell. Parlament, Parteien und "Staatsreform“ im Frankreich der Dritten und Vierten Republik, in: Von der Arbeiterbewegung zum modernen Sozialstaat. Festschrift für Gerhard A. Ritter zum 65. Geburtstag. Hg. von Jürgen Kocka, Hans-Jürgen Puhle und Klaus Tenfelde, München u.a. 1994, S. 409-428

Young, John W., Britain, France and the Unity of Europe 1945-1951, Leicester 1984

Young, Robert J., In Command of France. French Foreign Policy and Military Planning, 1933-1940, Cambridge/Mass. 1978

Ders., Power and Pleasure. Louis Barthou and the Third Republic, Montreal 1991

Ziebura, Gilbert, Léon Blum. Theorie und Praxis einer sozialistischen Politik. Band I: 1872 bis 1934, Berlin 1963

Ders., Die deutsch-französischen Beziehungen seit 1945. Mythen und Realitäten. Überarbeitete und aktualisierte Neuausgabe Stuttgart 1997

Zimmermann, Ludwig, Frankreichs Ruhrpolitik von Versailles bis zum Dawesplan, Göttingen 1971 


\section{Personenregister}

Der Name Reynaud, Paul wurde nicht in das Register aufgenommen.

Kursiv gesetzte Zahlen verweisen auf die Fußnoten der genannten Seite.

Abetz, Otto 302, 304, 347

Adenauer, Konrad 377, 382

Ageron, Charles-Robert 202

Alibert, Raphaël 340

Altorffer, Charles 90

Anglès, Raoul $76 \mathrm{f}$.

Appell, Paul 15, 16

Arago, François 80

Aron, Alexandre 84

Aron, Raymond 371,372

Artaud, Denise 101

Auburtin, Jean 254

Audiffret-Pasquier, Etienne d' 156

Auriol, Vincent $81 \mathrm{f} ., 86,151,152,358,360$

Badie, Vincent 315

Ballensiefen, Heinz 351

Bao-Dai 214

Bardoux, Jacques 308

Bardoux, Jean 54

Baréty, Léon 3

Bariéty, Jacques 100

Barodet, Désiré 75

Barrès, Maurice 92

Barthélemy, Joseph 17, 146

Barthou, Louis 3, 13, 30, 33, 53f., $57 \mathrm{f}$., 258f., 307

Bastid, Paul 371

Baudouin, Paul 320, 323, 325, $334 \mathrm{f} ., 342$

Becker, Jean-Jacques 59

Becker, Johann 105

Beneš, Edvard 62, 64, 302

Benoist, Charles 35, 46

Bérard, Léon 14

Bernard, Paul 209

Berstein, Serge 3

Beuve-Méry, Hubert 367

Bevin, Ernest 373

Bidault, Georges 357, 363-365, 367-369, 372,381
Billiet, Ernest 128

Binet-Valmer, Jean 113

Blanc, Balthazar 55

Blanc, Louis 26

Blum, Léon 10, 19, 21, 46f., 111, 129, 130 , 152 f., 163, 185 f., $192 \mathrm{f}$., 212, 220, 229, 238, $242,261,267,269-273,275-278,280,282$, $285,300,314$ f., 328, 343, 346f., 350, 351, $353,357,360,367-369$

Bokanowski, Maurice 14, 17

Bon, Jean 112

Bonnefous, Georges 59, 75, 79, 107, 129

Bonnet, Georges 156, 282, 298-301, 303306, 308

Bonnet, Henri 360

Bonnet, Joseph Louis 111

Borel, Berthe 16

Borel, Emile 16, 231

Boris, Georges 245, 253

Bouisson, Fernand 250

Boulanger, Georges 29, 41, 224

Bourdieu, Pierre 157

Bourgeois, Léon 38, 45, 142

Bourgeois, Louis 104

Bouthillier, Yves 251, 293, 325, 335

Bräuer, Curt 269

Braunias, Karl 218

Breitscheid, Rudolf 169

Brender, Reinhold $220 f$.

Briand, Aristide 17, 19, 39, 54, $56 \mathrm{f}$., 80, 82, 86f., 89-93, 101, 107, 109, 145, 153, 155, $157,160,168,171,174-176,178-181,183$, 185

Brinon, Fernand de 257

Brousse, Emmanuel 128

Bruce, David K. 360

Bülow, Bernhard Wilhelm von 169, 171

Bullitt, William C. 321,332

Buré, Emile 251

Busson-Billault, Julien-Henri 15 
Cachin, Marcel 116,121, 153

Caffery, Jefferson $359,368 f$.

Caillaux, Joseph $19,37,57,58$

Campbell, Sir Ronald 319f., 324, 329, 332, 340

Campinchi, César 54, 119, 246, 300, 306, 325

Capitant, René 223

Chamberlain, Arthur Neville 298-300, 303, 312,320

Chambrun, Charles Pineton de 66

Champetier de Ribes, Auguste 14,153, 280, $300 \mathrm{f}$.

Chappedelaine, Louis de 195

Charpentier, Jacques 351

Chatenet, Henri $231 \mathrm{f}$.

Chaumet, Jean-Charles 130

Chautemps, Camille 185, 245, 323, 325, $328-330,335,338,352$

Chauvel, Jean 337

Chauvineau, Louis 262

Chazelle, Jacques 368

Chéradame, André 150

Chéron, Henry 153, 181, 186f., $191 \mathrm{f}$.

Chiappe, Jean 217

Churchill, Sir Winston Spencer $300 \mathrm{f} ., 318 \mathrm{f}$., $323 \mathrm{f}$., 329, 330, $333 \mathrm{f}$., $340 \mathrm{f}$., 371, 376

Ciano, Galeazzo, Conte di Cortellazzo 306

Citroën, André 247

Claudel, Paul 371

Clemenceau, Georges 44, 64, 66-70, 72, 91, $183 \mathrm{f}$., 337,352

Clémentel, Etienne 184

Constant, Benjamin 34

Coquery-Vidrovitch, Catherine 202

Corbin, Charles 289, 320, 373

Cot, Pierre 346

Coty, François 118

Coty, René 20, 231

Coudenhove-Kalergi, Richard Nikolaus Graf von $371 \mathrm{f}$.

Courson, Léon 232

Courtin, René 371

Couve de Murville, Maurice 364

Cresson, Paul 54

Cromwell, Oliver 15

Cudenet, Gabriel 363

Curtius, Julius 185

Daladier, Edouard $19,21,61,132,145-147$, $153,156,161,184,214,236,245,256 \mathrm{f}$., $261,265-268,278,279,280-285,288,292$ 296, 298-309, 311-319, 321-324, 326, 334, $346 f ., 349 f ., 351,353,355,379,386$
Dariac, Adrien Louis 100

Darlan, François 321, 347

Daudet, Léon 92, 248

Dausset, Louis 139f., 142, 143

Dautry, Raoul 371

Déat, Marcel 219-221, 245 f., 253, 316

Debeney, Marie-Eugène 262

Debré, Michel 21, 41, 160,251,353, 364

Degoutte, Joseph 100

Dejean, Maurice 9,323, 325

Déroulède, Paul 46, 164, 224

Devaux, Gilbert 342

Diouf, Galandon 204

Dogan, Mattei 158

Doriot, Jacques 118

Doumer, Paul 16, 33

Doumergue, Gaston 20, 54, $57 \mathrm{f}$., 185, 202, 235-238, 242, 245, 250f., 252, 259, 286

Dreyfus, Alfred 14, 29, 30, 41, 43f., 51, 145

Dubois, Louis 151,154

Duclos, Jacques $19,110,112,113,116,121$, 139

Duguit, Léon 36

Dupuy, Charles 30

Durkheim, Emile 36

Duval-Arnould, Louis 129

Duverger, Maurice 368

Duvergier de Hauranne, Prosper 34

Eden, Robert Anthony 297, 316, 352

Ehrhardt, Hermann 165

Elwitt, Sanford 45

Engerand, Fernand 153

Erzberger, Matthias 98, 169

Esmein, Adhémar 35

Evain, Emmanuel 231

Fabre-Luce, Alfred 219

Fabry, Jean 3

Faure, Edgar 361

Faure, Paul 163

Ferry, Désiré 90

Ferry, Jules 26, 31, 41, 51, 131

Flandin, Pierre-Etienne 3, 19f., 33, 119, 128, $130,131,133,146,153,156,180,230,250$, $260,286,298,300,304$

Foch, Ferdinand 176, 183

Forgeot, Pierre 81 f., 86, 107,

Fournier, Albert 112, 116, 121, 139

François-Poncet, André 78, 118, 128, 130, 133, 135 f., 142, 146, 180, 186, 198, 199 , $258,368,379$

Frankenstein, Robert 282 
Franklin-Bouillon, Henri 3,117, 130-132, 150, 153, 176

Frey, Charles 90

Galimand, Lucien $315 f$.

Galliffet, Gaston de 30

Gambetta, Léon 13, 26-28, 35, $42 \mathrm{f}$.

Gamelin, Maurice 20, 22, 262, 264, 292, 311 , $319,321,323,332,346 \mathrm{f}$.

Gassier, Adrien 11, 55, 59

Gassier, Aimé 11,59

Gassier, Amélie 11

Gassier, Hippolyte 11

Gassier, Maurice 61

Gaulle, Charles de 20, 22 f., 47, 254 f., 259261, 262, 263-266, 280, 301, 308, 325, 332, $335-337,342,345,349,352$ f., 356, 357, $364,367,379,381-384,387$

Georges, Alphonse 262

Géraud, André [Pertinax] $351 \mathrm{f}$.

Germain-Martin (Martin, Germain) 247, 250

Geßler, Otto 168

Giraud, Emile 377

Giscard d'Estaing, Edmond 209

Giscard d'Estaing, Valéry 41

Goguel, François 59, 279

Gouin, Félix 357

Gourdeau, Gaston 232

Grévy, Jules 26

Grèzes-Rueff, François 157, 161

Gruet, Paul 224

Guernut, Henri 153

Guiter, Jean 129

Haguenin, Emile 78, 98

Halifax, Edward Frederick Lindley Wood, $3^{\text {rd }}$ Viscount of $298 \mathrm{f}$., $302 f$., 306, 316, $319 f$.

Harvey, Oliver 316, 321

Hauriou, Maurice 36

Hémery, Daniel 213

Henlein, Konrad 302

Henri-Robert (Robert, Henri) 15, 54

Henri-Robert, Jeanne (Erste Ehefrau Reynauds) 15, 158

Henri-Robert, Jeanne (Gattin Henri-Roberts) 15

Héraud, Marcel 13

Hermes, Andreas 102

Herriot, Edouard 19, 109, 145f., 151, 152, $153,173 \mathrm{f} ., 245,300,305,315,330,338$, $340,353,367,371,373,379$

Hervé, Gustave 164, 225
Hesnard, Oswald 165

Heydrich, Reinhard 267

Hilger, Gustav 347

Hirsch, Julius 94-97, 99

Hitler, Adolf 21, $255 \mathrm{f}$., 257, $258 \mathrm{f}$., 261, 279, 283, 295-299, 302-304, 306, 324

Hoesch, Leopold von 105, 169, 171, 178f., 181, $185 f$., 199

Hoffman, Paul 360

Honnorat, André 59, 60,73, 76

Hoover, Herbert Clark 250

Hudemann, Rainer 26

Hüser, Dietmar $364 f$.

Hugenberg, Alfred 163, 165

Hulin, Gaston 129

Hutin, Marcel 166, 196

Ignace, Edouard 111

Inghels, Albert 112

Irvine, William D. 4

Istel, André 251

Istel, Paul 14

Jackson, Julian 237f., 252

Janin, Maurice 63-69

Jardin, André 26

Jaurès, Jean 41, 265

Jeanneney, Jean-Noël 339

Jeanneney, Jules $301,305,306,308,309,313$, $317,321,330,336,338-341$

Jonnart, Charles 30

Joseph, Gaston $213 f$.

Jouvenel, Bertrand de 219, 245

Jouvenel, Henri de 218, 246

Kammerer, Albert 351

Kaufmann, Max R. 171

Kerillis, Henri de 18, 111-114, 116-123, 125-129, 133, 155f., 219, 226f., 245, 249, $252,253,304$

Kessel, Martina 364

Keynes, John Maynard 190

Klönne, Moritz 165-167, 171

Köhler, Heinrich 165

Köpke, Gerhard 167

Koltschak, Aleksandr W. 69

Kriegk, Otto $165 \mathrm{f}$.

Krumeich, Gerd 26, 59

Labarthe, André 349

Labori, Fernand 15

La Chambre, Guy 265, 306

Lachapelle, George 73,137, 138

Lacouture, Jean 353 
Lamoureux, Lucien 335

Laniel, Joseph 22, 246, 343, 356, 369

Lapie, Pierre-Olivier 363

La Rocque, François de 219, 237

Lasteyrie du Saillant, Charles de 100, 156

Laurent, Fernand 315

Laval, Pierre 19-21, 189, 199, 250, 253, 259, 286, 296, 340, 343, 347, 354

Lazareff, Pierre 314

Lebrun, Albert 22, 309, 313, 338, 341

Leca, Dominique 251, 322, 342

Le Corbeiller, Maurice 129

Ledru-Rollin, Alexandre 26

Léger, Alexis $300,335,352$

Le Gléo, Charles 119

Le Grix, François 19, 179

Lenoir, Jean-Louis 112

Leo XIII., Papst 49

Leopold III., König von Belgien 331

Leroy-Beaulieu, Paul 289, 291

Letailleur, Eugène 224

Le Trocquer, Yves 146

Leygues, Georges 86

Leyret, Henry 42, 43, 224

Lichtenberger, Henri 104, 106

Loucheur, Louis 78, 80, 86, 91

Ludendorff, Erich $98 \mathrm{f}$.

Mackensen, Hans Georg von 105

Mac-Mahon, Edme Patrice de 224

Maginot, André 128, 132, 186

Mahraun, Artur 165, 170

Maltzan, Adolf Georg Otto von, Freiherr zu Wartenberg und Penzlin $102 \mathrm{f}$.

Mamelet, Albert 130,131, 156

Mandel, Georges 153, 184, 280, $300 \mathrm{f} ., 306$, $313,325,337,341,347,350,351 f$.

Marchandeau, Paul 231, 235f., 282, $284 \mathrm{f}$., 290, 306

Margaine, Alfred 232

Margairaz, Michel 252, 282

Margerie, Pierre Jacquin de 165,171

Marie, André 22, 357 f., 360f., 369, 373

Marin, Louis 22, 75, 111, 127, 129, 131-133, 137, 151, 153-157, 297, 304, 314, 315, 322

Marjolin, Robert 374

Marquet, Adrien 220

Marrane, George 116

Martius, Georg 104

Marx, Karl 115

Marx-Dormoy (Dormoy, Marx) 345

Masaryk, Thomas 62f., 65

Mascuraud, Alfred 37, 74, 113

Mauriac, François 368
Maurin, Louis 262, 266

Maurras, Charles 44, 248

Mayer, René 358, 360, 376

Mayr, Karl 165, 169

Mazerand, Georges 90

Medem, Walter Eberhard von $165 \mathrm{f}$.

Méline, Jules 185

Mendès-France, Pierre 232, 246, 377

Menthon, François de 373

Mercier, Edmond 247

Millerand, Alexandre 3,13f., 18, 29, $85 \mathrm{f}$, $103,111,113 \mathrm{f} ., 116,121,125-129,132$, 169, 183, 185, 217, 224f., $229 \mathrm{f}$.

Mollet, Guy 373

Monicault, Pierre de 156

Monnet, Georges 314

Monnet, Jean 293, 361

Montagnon, Barthélémy 220

Montesquieu, Charles de Secondat, Baron de la Brède 52

Monzie, Anatole de 14, 307, 314, 324, $334 \mathrm{f}$.

Moreau, Félix 36

Mouré, Kenneth 252

Moutet, Marius 199

Müller, Klaus-Jürgen $113 f$.

Muller, Eugène 90

Mussolini, Benito 21, 276, 306, 324, 334

Mutius, Gerhard von $104 f$.

Napoléon Bonaparte 157

Napoléon III. 262

Noblemaire, Georges 81,82

Nogaro, Bertrand 245

Obregon, Alvaro 17

Ordioni, Pierre 367

Ormesson, Wladimir d' 314

Ozanam, Yves 12

Painlevé, Paul $16,118,145,168,171$

Palewski, Gaston 251, 364

Pasemann, Martin Ernst 351

Pasquier, Pierre 213, 214

Paté, Henri 112

Patenôtre, Raymond 219, 245f., 248, 253

Paul-Boncour, Joseph 245, 300

Payen, Henri 14

Pereboom, Maarten L. 171

Peretti de la Rocca, Emmanuel 106

Péri, Gabriel 304

Pertinax siebe Géraud, André

Pétain, Philippe 20, 22, 310, 323, 325, $327 \mathrm{f}$., $331,333,335,337,338,339-347,351 \mathrm{f}$., $354 \mathrm{f}$. 
Petsche, Maurice 361

Peyerimhoff, Henry de 97

Peytral, Victor 56,58

Philip, André 363, 369, 376

Philippoteaux, Henri 90

Phipps, Sir Eric 300, 302f., 306

Piétri, François 214

Pinay, Antoine 356, 376

Plaisant, Marcel 17, 79

Pleven, René 376

Poincaré, Raymond 13, 14, 18f., 30, 33, $42,47,57,91-94,97,99-107,108,129$, 136-138, 142, 146-151, 153-157, 160, $167,168,171-173,176,178,180,183-185$, 187,190 f., $193,218,222,278,284,356$, 361, 389

Pompidou, Georges 41

Portes, Hélène de 325, 342

Prouvost, Jean 351

Puech, Louis 112

Quaatz, Reinhold 170

Queuille, Henri 22, 357, $360 \mathrm{f}$.

Ramadier, Paul 234, 357, 371

Rathenau, Walther 86, 96

Ratier, Antony 130, 131, 156

Réache, Gerville 243

Rechberg, Arnold 19, 95, 98-106, 162, 164-166, 168-172, 177

Rechberg, Fritz 171

Reibel, Charles 14, 79, 107, 130

Reinach, Joseph 76

Renaitour, Jean-Michel 246

Renan, Ernest 354

Renaud, Jean 237

Reynaud, Albert 60

Reynaud, Alexandre 11f., 60

Rheinbaben, Werner von 165

Ribbentrop, Joachim von 267, 302, 305, 347

Ribot, Alexandre 13

Richelieu, Armand-Jean du Plessis, Herzog von 363

Richthofen, Hartmann von 165

Rieth, Kurt 163, $166 f$.

Rio, Alphonse 306

Rist, Charles 247

Ritter, Karl 165, 167

Rollin, Louis 231, 233

Roosevelt, Franklin Delano 198, 241, 307, $321,324,336$

Rosenberg, Frederic Hans von 103-105

Rostand, Edmond 15
Rothschild, Maurice de $81 \mathrm{f}$.

Rouvier, Maurice 185

Saint-Quentin, René de 324

Sanson, Rosemonde 4,32

Sarraut, Albert 137, 199, 202-204, 300, 306

Sarraut, Maurice 130, 199

Sartre, Jean-Paul 61

Sauerwein, Jules 170

Sauvy, Alfred 21, 241, 251, 272, 290

Scapini, Georges 246

Scheer, Charles 90

Scherer, Marc 363

Schmidt, Albert 104

Schneider-Creusot (Schneider, Eugène) 103, 169

Schubert, Carl von $165 f$., 167, 171

Schuman, Robert 90, $357 \mathrm{f} ., 369,375 \mathrm{f}$.

Schuschnigg, Kurt 296

Sellier, Louis 232

Sérot, Robert 90

Serre, Charles 329

Seydoux, Jacques $78,85,86$

Shirer, William L. 332

Siegfried, André 371

Sieyès, Emmanuel Joseph 226

Simon, John 308

Simon, Jules 26

Simonin, Camille 90

Sorlin, Pierre 26, 45, 49

Soulier, Edouard 79, 107, 129, 151

Spaak, Paul-Henri 371, 374

Spears, Edward L. 329, 340

Spofford, Charles M. 376

Stavisky, Alexandre 236

Steeg, Théodore 189, 211

Stefánik, Milan R. $62 \mathrm{f}$., $65 \mathrm{f}$.

Sternhell, Zeev 221

Stinnes, Hugo 84f., 101, 103-107, 169

Stone, Judith F. 45

Stresemann, Gustav 106, 162, 165 f., 171

Taittinger, Pierre 17, 79, 107, 111, 113, 116, 139, 219

Tardieu, André 3, 10,19-21, 80, 130, 134, 162, 175, 177-181, 183-199, 209, 211, $218 f$., 227, 229f., 233, 238, 249, 256, 279 , 281, 296, 388

Teitgen, Pierre-Henri 371, 376

Thiers, Adolphe 27, 177

Thorez, Maurice 261

Tirard, Paul 100, 106

Tixier, Adrien 352 
Tixier-Vignancourt, Jean-Louis 344

Touchard, Jean 40

Tournès, René 171

Treviranus, Gottfried Reinhold 165

Ullstein, Franz 165

Velpry, Pol-Maurice 262

Viénot, Pierre 164, 165, 172

Villelume, Paul de 292, 325, 334, $335 \mathrm{f}$.

Viviani, René $13 \mathrm{f}$.

Waldeck-Rousseau, René 13-15, 29-32, $38,41-52,54,58,142,161,183,276,278$, 385

Warren, Edouard de 79, 90,107

Weismann, Robert 165
Welczeck, Johannes Graf von 302, 304

Welles, Sumner $307 \mathrm{f}$., 324, 336

Wendel, François de 90, 97, 128, 151, $153 \mathrm{f}$, 156, 176, 247

Wendel, Humbert de 90

Weygand, Maxime 20, 22, 262, 323-325, $331,333 \mathrm{f} ., 335,337,339,343-345,351 \mathrm{f}$.

Wilson, Thomas Woodrow 64, $68 \mathrm{f}$.

Winkler, Heinrich August 220

Wirth, Joseph 87, $95 \mathrm{f}$.

Wulf, Peter $104 \mathrm{f}$.

Ybarnégaray, Jean 297f., 322

Zapf, Heinrich 96, 97

Zay, Jean 300, 306

Zola, Emile 42 